\title{
Maximum antigen diversification in a lyme bacterial population and evolutionary strategies to overcome pathogen diversity
}

\author{
Lia $\mathrm{Di}^{1,7}$, Saymon Akther (D) $^{2,7}$, Edgaras Bezrucenkovas ${ }^{1}{ }^{1}$, Larisa Ivanova ${ }^{3,6}$, Brian Sulkow ${ }^{1}$, Bing Wu ${ }^{1}$, Saad Mneimneh ${ }^{2,4}$, \\ Maria Gomes-Solecki (iD ${ }^{3}$ and Wei-Gang Qiu (iD ${ }^{1,2,5}$
}

(c) The Author(s), under exclusive licence to International Society for Microbial Ecology 2021

\begin{abstract}
Natural populations of pathogens and their hosts are engaged in an arms race in which the pathogens diversify to escape host immunity while the hosts evolve novel immunity. This co-evolutionary process poses a fundamental challenge to the development of broadly effective vaccines and diagnostics against a diversifying pathogen. Based on surveys of natural allele frequencies and experimental immunization of mice, we show high antigenic specificities of natural variants of the outer surface protein $\mathrm{C}(\mathrm{OspC})$, a dominant antigen of a Lyme Disease-causing bacterium (Borrelia burgdorferi). To overcome the challenge of OspC antigenic diversity to clinical development of preventive measures, we implemented a number of evolution-informed strategies to broaden OspC antigenic reactivity. In particular, the centroid algorithm - a genetic algorithm to generate sequences that minimize aminoacid differences with natural variants-generated synthetic OspC analogs with the greatest promise as diagnostic and vaccine candidates against diverse Lyme pathogen strains co-existing in the Northeast United States. Mechanistically, we propose a model of maximum antigen diversification (MAD) mediated by amino-acid variations distributed across the hypervariable regions on the OspC molecule. Under the MAD hypothesis, evolutionary centroids display broad cross-reactivity by occupying the central void in the antigenic space excavated by diversifying natural variants. In contrast to vaccine designs based on concatenated epitopes, the evolutionary algorithms generate analogs of natural antigens and are automated. The novel centroid algorithm and the evolutionary antigen designs based on consensus and ancestral sequences have broad implications for combating diversifying pathogens driven by pathogen-host co-evolution.
\end{abstract}

The ISME Journal (2022) 16:447-464; https://doi.org/10.1038/s41396-021-01089-4

\section{INTRODUCTION}

Antigen diversification driven by host-pathogen co-evolution Negative-frequency-dependent selection (NFDS) is an evolutionary mechanism that favors rare phenotypes over common ones, promoting biological novelty [1-3]. Driven by NFDS, antigenic variation is a molecular strategy widely shared among viral, bacterial, and eukaryotic pathogens to evade host immune defense [4-6]. Consequently, the power of NFDS in driving pathogen diversity becomes a fundamental challenge for developing broadly effective diagnostics and vaccines against fast-evolving microbial pathogens [7-9]. Although bacterial pathogens do not evolve as rapidly as viral pathogens, development of broadly effective diagnostics and vaccines is nonetheless hampered by a large number of cell-surface antigens as well as by the vast allelic diversity segregating at individual antigen loci in natural bacterial populations [5].

Here we hypothesize that, besides the structural and functional constraints to the relentless and seemingly random sequence diversification of microbial surface antigens, evolutionary rules govern antigen diversification as well. Specifically, we propose and test the hypothesis of maximum antigenic diversification (MAD) that co-existing antigen variants in a microbial population are obligatorily distinct from one other in antigenicity. The MAD hypothesis is a corollary of the strain theory of pathogen-host coevolution, which posits that host immunity drives pathogen populations into distinct genotypes ("strains") separated from one another by large genetic distances $[3,6]$. Under the strain model, co-existing pathogen strains occupy high-fitness peaks on an antigenic landscape shaped by host immunity where any off-peak antigen variants (e.g., recombinants) are at a selective disadvantage and would be eliminated by the host immunity [3]. This evolutionary rule may be exploited to tip the balance of the host-pathogen co-evolution for the benefit of the host. For example, the precarious coexistence of pathogen strains could be destabilized and the pathogen population be eliminated if the host immunological landscape is remolded by, e.g., an introduction of novel antigen variants as vaccines.

\section{Antigenic variations in Lyme disease pathogens}

For over three decades, Lyme disease has been the most prevalent vector-borne disease in the United States and Europe [10]. It is

\footnotetext{
${ }^{1}$ Department of Biological Sciences, Hunter College, City University of New York, New York, NY, USA. ${ }^{2}$ Graduate Center, City University of New York, New York, NY, USA. ${ }^{3}$ Department of Microbiology, Immunology and Biochemistry, University of Tennessee Health Science Center, Memphis, TN, USA. ${ }^{4}$ Department of Computer Science, Hunter College, City University of New York, New York, NY, USA. ${ }^{5}$ Department of Physiology and Biophysics \& Institute for Computational Biomedicine, Weil Cornell Medical College, New York, NY, USA. ${ }^{6}$ Present address: Pediatrics Department, New York Medical College, Valhalla, NY, USA. ${ }^{7}$ These authors contributed equally: Lia Di, Saymon Akther.

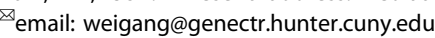


caused by spirochetes of the Borrelia burgdorferi sensu lato (Bbsl hereafter) species complex, also known as a new bacterial genus Borreliella $[11,12]$. A single species, $B$. burgdorferi sensu stricto $(B$. burgdorferi hereafter), transmitted by Ixodes scapularis ticks in the Northeast and Midwest and I. pacificus in the West, causes the majority of Lyme disease cases in the US. Genes encoding cellsurface lipoproteins are overrepresented in the $\sim 1.5 \mathrm{Mbp}$ genome of $B$. burgdorferi, totaling $4.9 \%$ of the chromosomal genes and $14.5 \%$ of the plasmid-encoded genes, in contrast to $2.0 \%$ lipoprotein-encoding genes in other bacterial pathogens such as Helicobacter pylori and Treponema pallidum [13]. Genome comparisons further revealed that lipoprotein-encoding genes are the most variable loci within the genome, consistent with their roles in evading vector and host immunity [14]. Specifically, $B$. burgdorferi modulates cell-surface lipoprotein composition when migrating between the tick vector and the mammalian host. For example, the expression of the outer surface protein A (OspA) is downregulated within a mammalian host while the expression of the outer surface protein $\mathrm{C}(\mathrm{OspC})$ is upregulated during host invasion and, subsequently, the spirochete cells generate and express genetic variants at the vls (variable membrane protein-like sequences) locus to enable persistent infection of the host [15-17].

Driven by diverse forms of natural selection and with distinct cellular functions, Bbsl surface antigens differ in the rate and pattern of sequence evolution [14, 18-20]. For example, DNA sequences encoding OspA vary little among strains of the same $B b s /$ species while differing greatly among the Bbsl species [21]. Genes encoding OspC display high sequence variability within as well as between the Bbsl species as a result of diversifying selection [22-25]. The silent cassettes at the vls locus vary greatly between-species, within-species, as well as during the course of infection as a result of host adaptive immunity [17, 26-30].

Among the large repertoire of genes encoding cell-surface lipoproteins in Bbsl, ospC plays an outsized role in evading host immunity and establishing infection. First, ospC is required for the initial invasion into the host, suggesting its role in defense against host innate rather than adaptive immunity [31, 32]. Host cellular and molecular targets of OspC remain to be identified, although it has been shown that ospC expression was associated with the anti-phagocytosis and plasminogen-binding activities of the spirochete [33, 34].

Second, OspC is the immunodominant and serotype-determinant antigen of Bbs/ strains [24, 35]. Experimental immunization of mice with recombinant OspC variants elicited strain-specific protective immunity against strains expressing homologous but not heterologous OspC variants [36-39]. Further, experimental immunization of mice using whole sera from infected mice showed that polyclonal antibodies binding OspC were the main components of strainspecific immunity [39]. Field-based studies further supported NFDS acting on the ospC locus being the main evolutionary mechanism maintaining genomic diversity in natural $B$. burgdorferi populations $[22,40-42]$.

Third, sequence variations at ospC are in nearly complete linkage disequilibrium with genomic lineages in North America, suggesting that the within-population $B$. burgdorferi strain diversification is driven by $\operatorname{ospC}$ variability $[40,43]$. Simulations based on principles of population genetics showed that the nearly one-to-one correspondence between the major ospC alleles and the co-existing $B$. burgdorferi lineages was consistent with a history of within-population genome diversification driven by NFDS targeting the $o s p C$ locus [40]. Additional evidence supporting the ospC-driven diversification of $B$. burgdorferi strains includes the high recombination rate at $\operatorname{ospC}$ and the uniform distributions of ospC alleles [23, 42]. While it remains a possibility that sequence variation at $\operatorname{cspC}$ is associated with host diversity in this generalist parasite [25], the "multiple-niche" hypothesis appears to be inconsistent with results of field studies of $B$. burgdorferi populations in North America and B. afzelii populations in Europe as well as with results of experimental investigations [44, 45].

\section{Quest for broadly cross-reactive OspC molecules}

Immuno-dominance of OspC makes it a valuable target for antiLyme diagnostics and vaccines, yet its clinical potentials are limited by its sequence hyper-variability. Thus far, strategies to overcome OspC diversity included identification of conserved epitopes or variable epitopes distinct among natural variants [46-48]. However, conserved sequences and domains on the OspC molecule were ineffective targets of vaccination [46, 49]. In an high-throughput investigation, key OspC epitopes were mapped to the hypervariable C-terminal region with the use of protein arrays and sera from mice and humans [47]. A minimum set of OspC variants had been identified as candidates of broadly effective diagnostics on the basis of quantifying the antigenic breadth of OspC variants with the use of sera from immunized mice as well as sera from naturally infected hosts [48]. A concatenation of eight OspC epitopes associated with distinct natural variants were the base of a broadly immunogenic vaccine for canine use $[46,50]$.

The MAD hypothesis suggests an alternative and novel strategy to overcome the limitation of OspC sequence diversity to the development of OspC-based diagnostics and vaccines. First, on the basis of frequency distributions of antigen variants in nature and experimental immunization of mice, here we tested MAD among the $16 \mathrm{OspC}$ variants co-existing in natural populations of the Lyme disease pathogens in the Northeast United States $[40,51]$. Second, we used evolutionary algorithms to design analogs of natural OspC molecules with minimized sequence differences to the 16 natural variants. We cloned and purified these synthetic OspC molecules and tested their antigenic breadths using sera from artificially and naturally infected hosts. Third, we explored molecular mechanisms underlying the broad antigenicity of evolutionary antigens with a mathematic model and computer simulations. One of our evolution-based designsthe consensus algorithm-was similar to the COBRA approach used to design broadly reactive vaccines against the influenza virus [8]. The root algorithm-another evolution-informed algorithm implemented in the present study-has been used to design vaccine candidates against diverse strains of the human immunodeficiency virus type I (HIV-1) [52]. Critically, these evolution-based strategies are automated and able to generate synthetic analogs that assume the structure, function, and epitope configurations similar to those of the native antigens while displaying broader antigenicity.

\section{MATERIALS AND METHODS \\ Co-occurrences of OspC variants in field-collected Ixodes ticks and a test of OspC specificity}

We tested the immunological distinction of $B$. burgdorferi strains based on their co-occurrences in individual Ixodes scapularis ticks. Deep highthroughput sequencing of individual nymphal ticks, which had fed a single blood meal as larvae, revealed that multiple $B$. burgdorferi strains within a single tick were caused mainly by the mixed infection of the host rather than by a history of feeding on multiple hosts [23, 53]. As such, we hypothesized that immunologically distinct strains tended to co-infect a single tick while immunologically similar strains tended to be found in different ticks. We tested the hypothesis with a previously published data set that recorded the presence and absence of 20 Lyme pathogen strains within $n=119$ I. scapularis ticks collected from New York State during 2015 and 2016 [54] (Supplementary Information Data S1). While the data set consisted of mostly adult ticks with only 25 nymphal ticks, there were no significant differences in the level of $B$. burgdorferi strain diversity carried by single ticks among the nymphal, adult male, and adult female ticks [23]. In other words, the individual infected nymphal ticks carried B. burgdorferi strains as diverse as the adult ticks despite an additional blood meal the adult ticks have taken. In a separate study using high-throughput sequencing of infected nymphal ticks from the same region, the authors 


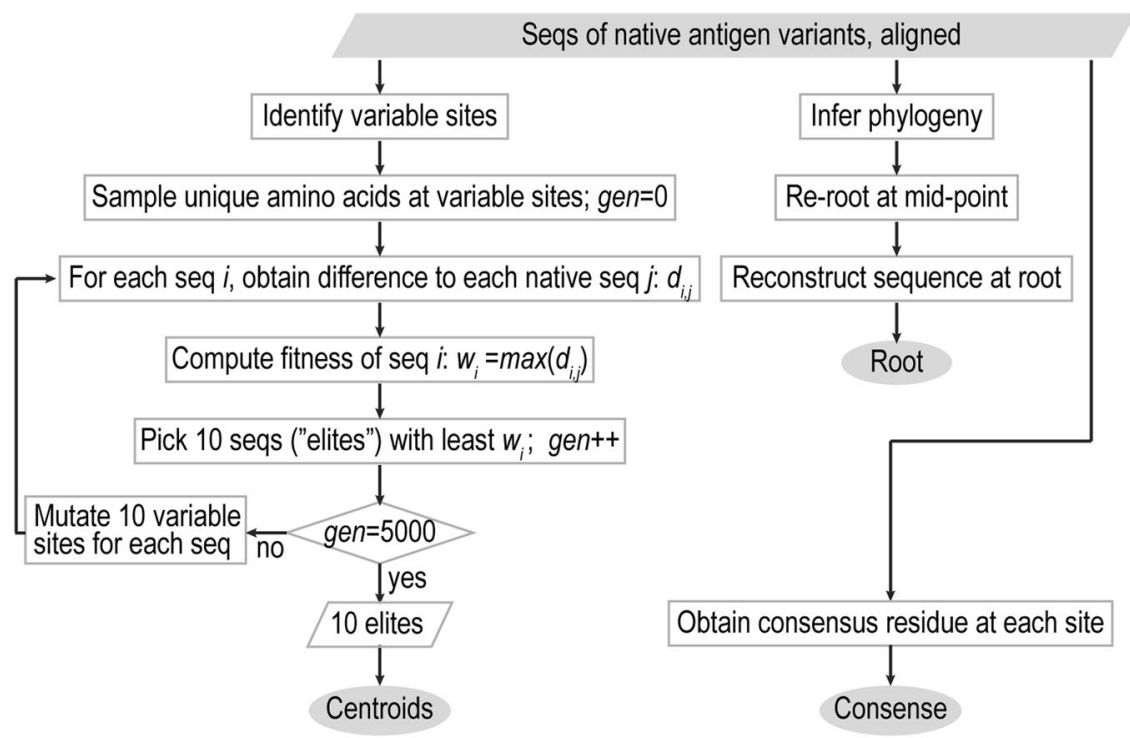

Fig. 1 Evolutionary algorithms for the design of broadly reactive OspC antigens. The centroid algorithm used a genetic algorithm to search for antigen sequences with minimized differences to the natural OspC variants ("centroids"). The root algorithm inferred an antigen sequence representing the mid-point root of a phylogenetic tree of the natural OspC variants ("root"). The consensus algorithm generated an antigen sequence consisting of majority amino-acid residues at individual positions of an alignment of the natural OspC sequences ("consense"). These evolutionary algorithms produced OspC analogs with approximately half the sequence differences to the natural variants than the distances among the natural variants themselves.

have similarly concluded that reservoir hosts were commonly infected by diverse $B$. burgdorferi strains [53]. Here, we quantified the over- or underabundance of a pair of strains ( $i$ and $j$ ) as the fold change of the observed over the expected counts: $F C(i j)=\log _{2}\left\{\frac{O b s(i j)}{\exp (i j)}\right\}$. Statistical significance of the relative abundance was obtained from a null distribution generated by permuting the occurrences of a pair of strains among infected ticks 1000 times while keeping the total occurrence constant. Significantly overor underrepresented pairs of $B$. burgdorferi strains co-infecting single I. scapularis ticks would suggest an absence or presence, respectively, of immune cross-protection for the strain pair.

Approximately $20 \mathrm{OspC}$ variants commonly coexist in B. burgdorferi populations in the Northeast US $[22,23,48]$. We aligned the protein sequences of 16 common natural OspC variants (named as " $\mathrm{A}$ " through "N", "T", and "U") with the program muscle [55] (Supplementary Information Text S1). Pairwise sequence differences among the OspC variants were calculated with the alignment utility bioaln from the BpWrapper software suite, which was based on BioPerl $[56,57]$.

\section{Evolutionary algorithms for designing broadly reactive synthetic OspC}

Protein sequences analogous to natural OspC variants were optimized for broad reactivity using three evolutionary algorithms (Fig. 1). By generating $\mathrm{OspC}$ analogs close to the root of a molecular phylogeny with natural OspC variants, these evolutionary algorithms aimed to reduce the sequence difference between an evolutionary analog with the natural variants to be approximately half of the difference among the natural variants themselves. The initial input for all three algorithms was the aligned amino-acid sequences of $16 \mathrm{OspC}$ variants (Supplementary Information Text S1). First, we inferred the hypothetical ancestral sequence at the midpoint root of the phylogeny of the natural OspC variants with RAxML [58] (the "Root Algorithm"). Second, we obtained a consensus sequence consisting of $20 \%$ majority residues at aligned sequence positions of the natural OspC variants with the consensus method implemented in the Bio:: SimpleAlign module of the BioPerl library [57] (the "Consensus Algorithm").

Third, we used a genetic algorithm to generate sequences with minimal distances to natural OspC variants (the "Centroid Algorithm"). Briefly, we extracted amino acids at variable positions from 16 aligned natural OspC sequences. An initial seed population of random antigen sequences $(n=$ 10) were generated through sampling the unique amino acids present at each variable site with uniform probabilities. For each randomly generated sequence $i$, we calculated its differences $\left(d_{i j}, j=\right.$ " $\mathrm{A}$ " through " $\mathrm{U}$ ") to the 16 natural variants. We defined the fitness of this sequence as the maximum value among its differences to all 16 natural sequences: $w_{i}=\max \left(d_{i j}\right)$. This fitness measured its overall sequence similarity to the natural variants-the lower the $w_{i}$ the higher its overall similarity to the natural variants. The top ten most similar antigen sequences ("elites") in each generation were retained and others were discarded. Each elite sequence was then allowed to "reproduce" 10 times with mutations at randomly selected ten variable sites, resulting in ten mutated "gametes". The above process was repeated (e.g., for 5000 generations) to progressively lower the $w_{i}$ values, after which the final output included ten elite centroids that were the most similar to all 16 natural OspC variants. The centroid algorithm was implemented with the BioPerl library in Perl [57] and the DEAP package in Python [59]. The top four optimized centroid sequences were cloned, overexpressed, and purified for immunological assays of antigenic breadth.

\section{Gene synthesis, protein overexpression, and protein purification}

DNA sequences encoding the natural and synthetic OspC variants were codon-optimized, synthesized, and cloned into the pET24 plasmid vector, which was then used to transform the Escherichia coli BL21 cells. All DNA work was performed by a commercial service (Genelmmune Biotechnology Corp., Rockville, MD, USA). We designed the OspC constructs by excluding the first 18 residues encompassing the signal peptide and by adding a $10 \times$ Histidine-tag on the $\mathrm{N}$-terminus. These modifications were necessary for overexpression of OspC proteins in E.coli and to facilitate OspC purification [60].

For each $E$. coli strain containing a cloned $\operatorname{csp} C$ gene, a single colony was selected to inoculate $4 \mathrm{ml}$ Luria-Bertani (LB) broth (Thermo Fisher Scientific Waltham, MA, USA) containing vector-specific selective antibiotics $(25 \mu \mathrm{g} /$ $\mathrm{ml}$ for Kanamycin or $50 \mu \mathrm{g} / \mathrm{ml}$ for Ampicillin). The seeded culture was incubated overnight at $37^{\circ} \mathrm{C}$ with vigorous shaking (250 rpm). A portion of the overnight culture was transferred into $50 \mathrm{ml}$ fresh pre-warmed LB broth containing $0.4 \%$ glucose and the selective antibiotics. The culture was incubated at $37^{\circ} \mathrm{C}$ with vigorous shaking until reaching exponential growth indicated by an $\mathrm{OD}_{600}$ of $\sim 0.8$ as measured by the NanoDrop Spectrophotometer (Thermo Fisher Scientific, Waltham, MA, USA). Expression of the cloned $0 s p C$ was induced by adding isopropyl $\beta$-d-1thiogalactopyranoside (IPTG) to a final concentration of $0.25-0.5 \mathrm{mM}$ and by incubation overnight at $25^{\circ} \mathrm{C}$. Cells were collected by refrigerated centrifugation at $4^{\circ} \mathrm{C}$ and $7200 \mathrm{rpm}$ for $15 \mathrm{~min}$, resuspended in a lysis buffer containing $0.2 \mathrm{mg} / \mathrm{ml}$ lysozyme, $20 \mathrm{mM}$ Tris- $\mathrm{HCl}(p \mathrm{H} 8.0), 250 \mathrm{mM}$ $\mathrm{NaCl}$, and $1 \mathrm{mM}$ dithiothreitol (DTT). After incubation for $1 \mathrm{~h}$ at $4{ }^{\circ} \mathrm{C}$, cells were further lysed by sonication until the solution become translucent. The lysate was centrifuged in refrigeration at $12,000 \mathrm{rpm}$ for $20 \mathrm{~min}$ and the supernatant was withdrawn. 
The recombinant proteins were purified using nickel sepharose beads (Ni-NTA, Thermo Fisher Scientific, Waltham, MA, USA). The lysate supernatant from the $50 \mathrm{ml}$ culture was mixed with $300 \mu \mathrm{l} \mathrm{Ni-NTA}$ beads and incubated overnight at $4{ }^{\circ} \mathrm{C}$ in the lysis buffer supplemented with $5 \mathrm{mM}$ imidazole. The lysate-bead mixture was then loaded into a chromatography column and washed with 12 times the bed volume of the lysis buffer containing $25 \mathrm{mM}$ imidazole. The purified protein was eluted with 6 times the bed volume of the lysis buffer containing $500 \mathrm{mM}$ imidazole. The elution was dialyzed to remove imidazole in phosphate-buffered saline (PBS, $p \mathrm{H}$ 7.4) containing $1 \mathrm{mM}$ DTT and $20 \%$ glycerol.

The amount and purity of recombinant proteins were examined using the sodium dodecyl sulfate polyacrylamide gel electrophoresis (SDS-PAGE) containing $12 \%$ gel following the standard protocol. The PageRuler Prestained 10-180 kDa Protein Ladder (Thermo Fisher Scientific, Waltham, MA, USA) was used to mark molecular weights. The gel was stained in $0.08 \%$ Coomassie Blue and de-stained in $45 \%$ methanol and $10 \%$ acetic acid. Concentration of the final purified protein solution was quantified using the Pierce Bradford Protein Assay Kit (Thermo Fisher Scientific, Waltham, MA, USA).

\section{Sera from naturally infected hosts and immunized mice}

The majority of human serum samples were provided by the Centers for Disease Control and Prevention (CDC) (Table 1). The human sera originated from patients diagnosed with early to late Lyme disease or from healthy individuals in endemic and non-endemic regions in the USA [61]. The CDC sera panel was previously screened using the standard two-tiered testing (STTT) for the presence of antibodies against B. burgdorferi, including $\operatorname{lgM}$, IgG, or both antibodies against OspC (the $23 \mathrm{kD}$ band) [62]. The CDC sera panel was custom compiled for the present study. Ten serum samples from Lyme disease patients were originally collected by the Stony Brook University Health Science Center, NY, USA. Ten serum samples were obtained from the natural reservoir of $B$. burgdorferi, the white-footed mouse (Peromyscus leucopus) from Milbrook, NY, USA. The latter human and mouse sera were screened for exposure to $B$. burgdorferi using the C6 ELISA (Immunetics, Boston, MA, USA).

Sixteen recombinant OspC were previously cloned from B. burgdorferi strains into the pET9c plasmid [48]. The proteins were expressed in E. coli BL21 (DE3) pLysS and purified under native conditions by ion exchange chromatography using Q-Sepharose Fast Flow (GE Healthcare, Sweden) as described previously [63]. C3H/HeJ mice (Mus musculus) and white-footed mice (P. leucopus) were immunized with $10-20 \mu \mathrm{g}$ of each of the 16 individual purified natural recombinant OspC proteins. Briefly, mice received a dose of recombinant protein on Day 1 and Day 14, and on Day 28 they were euthanized and blood collected by heart puncture. Animal experimentation followed the protocols approved by the Animal Care and Use Committee of University of Tennessee Health Science Center.

\section{Immunological assays}

Immunoblot assays of OspC variant-specific sera were performed using a MiniSlot/MiniBlotter 45 system (Immunetics, Boston, MA, USA). Briefly, a PVDF membrane (Millipore, Billeirca, MS, USA) was mounted on the MiniSlot and $25 \mu \mathrm{g}$ of each purified protein was loaded individually into its parallel channels. The proteins were immobilized onto the PVDF membrane after the excess solution was removed by vacuum aspiration, resulting in a deposit of horizontal parallel stripes of antigens. The membrane was released from the Minislot and blocked in 10\% skim milk (Difco, Sparks, MD, USA) for $2 \mathrm{~h}$ at room temperature. After blocking the membrane was rotated by 90 degrees and placed in the MiniBlotter 45 . Diluted mouse serum (1:100 to 1:1000 in 3\% of milk in TBS buffer with $0.5 \%$ Tween 20,150 $\mathrm{\mu l}$ ) was deposited in the individual vertical lanes of the Miniblotter and was incubated for $1 \mathrm{~h}$ at room temperature. The membrane was washed three times with TBS containing $0.5 \%$ Tween 20 and was incubated with goat anti-mouse IgG conjugated with alkaline phosphatase (1:2000) (Kirkegaard \& Perry Laboratories [KPL], Gaithersburg, MD, USA) for $1 \mathrm{~h}$ at room temperature. The BCIP/NBT Phosphatase Substrate (KPL) was used to visualize the signal. Serum of non-immunized mice and the bovine serum albumin were used as the negative serum control and the non-OspC antigen control, respectively. Binding intensity values were digitalized with ImageJ [64].

Sera from naturally infected hosts were tested for reactivity with the purified recombinant OspC proteins (rOspCs) through enzyme-linked immunosorbent assay (ELISA). Specifically, a 96-well MICROLON 600 plate (USA Scientific, Inc., Ocala, FL, USA) was loaded in each well with $100 \mu$ I PBS containing $10 \mu \mathrm{g} / \mathrm{ml}$ of a rOspC and incubated overnight at $4^{\circ} \mathrm{C}$. The coated plate was washed three times using PBS containing $0.1 \%$ Tween 20
(PBS-T buffer) and blocked with $200 \mu \mathrm{l}$ PBS-T buffer containing 5\% milk for $1 \mathrm{~h}$ at $37^{\circ} \mathrm{C}$. After washing three times with the PBS-T buffer, $100 \mu \mathrm{l}$ serum sample diluted in PBS by a factor between 1:100 to 1:1000 was added to each well and incubated for $1 \mathrm{~h}$ at $37^{\circ} \mathrm{C}$. After washing three times with the PBS-T buffer, $100 \mu \mathrm{l}$ diluted horseradish peroxidase (HRP)-conjugated secondary antibodies was added to each well. We used the Goat AntiHuman IgG/lgM $(\mathrm{H}+\mathrm{L}$ ) (Abcam, Cambridge, UK) diluted by a factor of 1:50,000 for assays of human sera and the Goat Anti-Peromyscus leucopus $\lg \mathrm{G}(\mathrm{H}+\mathrm{L})$ (SeraCare Life Sciences, MA, USA) diluted by a factor of 1:1000 for assays of $P$. leucopus sera. After incubation for $1 \mathrm{~h}$ at $37^{\circ} \mathrm{C}$ and washing with PBS-T buffer, $100 \mu \mathrm{l}$ TMB ELISA Substrate Solution (Invitrogen eBioscience) was added. The enzyme reaction proceeded for 15-30 min at room temperature and was terminated with $1 \mathrm{M}$ sulfuric acid. Binding intensities were measured at the $450 \mathrm{~nm}$ wavelength using a SpectraMax i3 microplate reader (Molecular Devices, LLC, CA, USA).

\section{Statistical analysis of OspC cross-reactivity}

We tested antigenic specificity of natural OspC variants by performing a reanalysis of a data set generated from a previously published study [48]. The data set consisted of replicated ELISA readings of the reactivity between the 16 recombinant OspC variants with the antisera $(n=15)$ of $\mathrm{C} 3 \mathrm{H} / \mathrm{HeJ}$ mice (Mus musculus) artificially immunized with the purified OspC variants (Supplementary Information Data S2). Sera from uninfected mice were used as the negative control.

To quantify the antigenicity of $\mathrm{OspC}$ variants with variant-specific mouse sera, we first transformed the raw $\mathrm{OD}_{450}$ readings and the digitalized binding intensities into normalized $z$-scores: $z_{r s}=\frac{X_{r s}-\text { Mean }\left(x_{s}\right)}{S D}$, where $x_{r s}$ is the binding value $\left(\mathrm{OD}_{450}\right.$ reading or binding intensity) of the recombinant OspC $r$ with the serum $s$ while Mean $\left(x_{s}\right)$ and $\mathrm{SD}\left(x_{s}\right)$ represent, respectively, the mean and the standard deviation of $\mathrm{OD}_{450}$ readings of all recombinant OspC variants with respect to the serum $s$. The rescaling was necessary to account for the systematic differences among the variant-specific sera in non-specific, background bindings due to e.g., the varying amount of total antibodies in a particular mouse serum. For example, the $\mathrm{OD}_{450}$ readings of variants-regardless homologous or heterologous antigens-with the variant K-specific serum were consistently higher than other sera $\left(\mathrm{OD}_{450}>\right.$ 1.0) in the original study [48] (Fig. 1 therein). Normalization with the $z$-scores made it possible to compare the reactivity of an OspC variant across the serum samples by reducing serum-specific background noise. Furthermore, a score of $z<-2.0$ or $z>2.0$ indicated a statistically significant (with $p=0.05$ ) deviation from the mean reactivity $(z=0)$ of an OspC variant with the variant-specific sera. Normalization of ELISA readings with $z$-scores had been used to reduce serum-specific background noises in a clinical diagnostic test [65].

To quantify the antigenicity of OspC variants with the naturally infected human and mouse sera, we first displayed the raw $\mathrm{OD}_{450}$ readings with bar graphs. To render the binding values comparable among the serum samples, we then transformed the $\mathrm{OD}_{450}$ values with respect to sera into $z$-scores as described above. The median value for a variant was a measure of its antigenic breadth, with a relatively high median indicating a relatively broad antigenicity. In addition, the transformed binding scores were visualized with heatmaps, which further identified hierarchical clusters of OspC variants and sera according to the similarities in binding levels. The $\mathrm{R}$ package pheatmap was used to generate the heatmaps.

To summarize the antigenic breadth of an OspC variant across the serum samples, we designed a novel measure of total antigen reactivity. The antigenic reaction characteristic (ARC) of an OspC variant was defined as a curve of cumulative binding values (with scaled $\mathrm{OD}_{450}$ readings) over the cumulative number of serum samples. A highly specific antigen variant would show as a low-lying ARC curve because of the consistently low $(z<$ 0 , below-average) binding values, with the exception of the high $(z>0$, above-average) binding values with the homologous anti-sera. A nonreactive antigen (e.g., a negative control) would generate all negative scores and show as a low-lying, monotonically decreasing curve. A broadly reactive antigen variant, in contrast, would show as an elevated ARC curve indicating consistently high $(z>0)$ bindings with sera. As such, the area under an ARC curve (AUC) corresponds to a higher cross-reactivity (or Tower specificity) of an OspC variant. The ARC curve is inspired by the receiver operating characteristic curve, which quantifies the specificity of a classification scheme or a diagnostic test by plotting the cumulative number of true positives against the cumulative number of false positives [66]. Statistical significance of an ARC curve (or an AUC) could be evaluated by comparing it with a distribution of ARC curves (or AUC values) 
Table 1. Serum samples.

\begin{tabular}{|c|c|c|c|c|c|c|c|}
\hline \multirow[t]{2}{*}{ Label } & \multirow[t]{2}{*}{ Host } & \multirow[t]{2}{*}{ Source } & \multirow[t]{2}{*}{ Description } & \multicolumn{3}{|c|}{ STTT Interpretation ${ }^{\mathbf{b}}$} & \multirow[t]{2}{*}{ C6 ELISA } \\
\hline & & & & EIA & $\lg M 23$ kD band & $\begin{array}{l}\text { IgG } 23 \\
\text { kD band }\end{array}$ & \\
\hline S01 & Human & $\mathrm{CDC}$ & $\mathrm{EM}^{\mathrm{a}}$ convalescence & + & + & + & $N A^{d}$ \\
\hline S03 & Human & $\mathrm{CDC}$ & EM convalescent & + & + & + & NA \\
\hline SO4 & Human & $\mathrm{CDC}$ & Non-endemic control & - & + & - & NA \\
\hline S05 & Human & $\mathrm{CDC}$ & Non-endemic control & - & - & - & NA \\
\hline S14 & Human & $\mathrm{CDC}$ & Fibromyalgia (control) & - & - & - & NA \\
\hline S16 & Human & $\mathrm{CDC}$ & Severe periodontitis (control) & - & - & - & NA \\
\hline S18 & Human & $C D C$ & EM convalescent & + & + & + & NA \\
\hline S21 & Human & $C D C$ & Endemic control & - & + & - & NA \\
\hline T04 & Human & $\mathrm{CDC}$ & EM & $\mathrm{Equ}^{\mathrm{e}}$ & - & - & NA \\
\hline T05 & Human & $\mathrm{CDC}$ & Lyme arthritis & + & + & + & NA \\
\hline T06 & Human & $\mathrm{CDC}$ & EM & + & + & + & NA \\
\hline T07 & Human & $\mathrm{CDC}$ & EM & Equ & + & - & NA \\
\hline T08 & Human & $C D C$ & EM & + & + & + & NA \\
\hline T09 & Human & $\mathrm{CDC}$ & EM & - & - & + & NA \\
\hline T10 & Human & $\mathrm{CDC}$ & EM & - & - & - & NA \\
\hline T11 & Human & $C D C$ & EM & + & + & + & NA \\
\hline $\mathrm{T} 12$ & Human & $\mathrm{CDC}$ & Lyme arthritis & + & - & + & NA \\
\hline T13 & Human & $C D C$ & Neurological Lyme & + & + & + & NA \\
\hline T20 & Human & $C D C$ & EM & + & + & + & NA \\
\hline T21 & Human & $\mathrm{CDC}$ & Neurological Lyme & - & + & + & NA \\
\hline T22 & Human & $C D C$ & EM & + & + & - & NA \\
\hline T23 & Human & $\mathrm{CDC}$ & EM & - & - & + & NA \\
\hline T24 & Human & $\mathrm{CDC}$ & EM & + & + & + & NA \\
\hline T25 & Human & $C D C$ & EM & + & + & + & NA \\
\hline T26 & Human & $\mathrm{CDC}$ & EM & - & + & - & NA \\
\hline T27 & Human & $C D C$ & EM & + & - & + & NA \\
\hline T29 & Human & $\mathrm{CDC}$ & EM & + & + & + & NA \\
\hline Т30 & Human & $\mathrm{CDC}$ & Neurological Lyme & + & + & + & NA \\
\hline T31 & Human & $C D C$ & EM & - & - & - & NA \\
\hline T32 & Human & $\mathrm{CDC}$ & Cardiac Lyme & + & + & + & NA \\
\hline H01 & Human & Stony Brook & Late Lyme & NA & NA & NA & 0.924 \\
\hline H04 & Human & Stony Brook & Late Lyme & NA & NA & NA & 1.947 \\
\hline H07 & Human & Stony Brook & Late Lyme & NA & NA & NA & 0.555 \\
\hline H08 & Human & Stony Brook & Late Lyme & NA & NA & NA & 0.260 \\
\hline H09 & Human & Stony Brook & Late Lyme & NA & NA & NA & 0.013 \\
\hline $\mathrm{H} 10$ & Human & Stony Brook & Late Lyme & NA & NA & NA & 0.491 \\
\hline P01 & P. leucopus & Millbrook, NY & Field reservoir & NA & NA & NA & 0.592 \\
\hline
\end{tabular}


Table 1 continued

\begin{tabular}{|c|c|c|c|c|c|c|c|}
\hline \multirow[t]{2}{*}{ Label } & \multirow[t]{2}{*}{ Host } & \multirow[t]{2}{*}{ Source } & \multirow[t]{2}{*}{ Description } & \multicolumn{3}{|c|}{ STTT Interpretation ${ }^{b}$} & \multirow[t]{2}{*}{ C6 ELISA ${ }^{c}$} \\
\hline & & & & EIA & IgM 23 kD band & $\begin{array}{l}\text { IgG } 23 \\
\text { kD band }\end{array}$ & \\
\hline P02 & P. leucopus & Millbrook, NY & Field reservoir & NA & NA & NA & 0.266 \\
\hline P03 & P. leucopus & Millbrook, NY & Field reservoir & NA & NA & NA & 3.480 \\
\hline P04 & P. leucopus & Millbrook, NY & Field reservoir & NA & NA & NA & 0.749 \\
\hline P05 & P. leucopus & Millbrook, NY & Field reservoir & NA & NA & NA & 0.910 \\
\hline P08 & P. leucopus & Millbrook, NY & Field reservoir & NA & NA & NA & 3.046 \\
\hline P09 & P. leucopus & Millbrook, NY & Field reservoir & NA & NA & NA & 0.518 \\
\hline P10 & P. leucopus & Millbrook, NY & Field reservoir & NA & NA & NA & 0.286 \\
\hline
\end{tabular}

${ }^{a} E M$ erythema migrans, an early-stage Lyme disease.

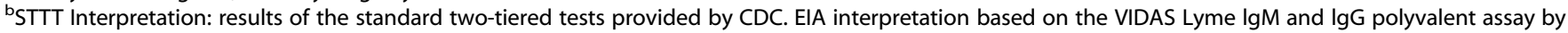
bioMerieux, Inc, with the following cutoff values: negative $<0.75$, equivocal $>0.75$ to $<1.00$, and positive $>1.00$. IgM and IgG immunoblotting assays by MarDx Diagnostics, Inc.

${ }^{c}$ C6 ELISA: OD450 readings from ELISA using the $C 6$ peptide.

${ }^{\mathrm{d}} N A$ not available.

equ equivocal.

generated with random permutations of the scaled $\mathrm{OD}_{450}$ readings among the same set of serum samples.

\section{A binary model and computer simulations of maximum antigen separation}

To explore immunological and molecular mechanisms underlying the broad antigenicity of evolutionary centroids, we construct a mathematical model and computationally simulated maximum antigen diversification (MAD) and evolutionary centroids. Following the multiple-epitope extension of the Gupta et al. model [3], we represented antigen variants in a pathogen population as binary strings.

In generating binary strings, let $p$ be the probability of the binary state 1 (and $1-p$ the probability of the binary state 0 ). Without loss of generality, we may assume that $p \leq 0.5$ since, otherwise, switching the roles of $0 \mathrm{~s}$ and $1 \mathrm{~s}$ preserves all distances (we use the Hamming distance) and changes $p$ to $1-$ $p$. Following the convention of using zeroes to represent ancestral states in evolutionary analysis, we designate the zero string (the string with all bits set to 0) as a "centroid". Mathematically, this choice of the centroid is general because flipping all bits in a given position preserves all distances. Two random binary strings of length $n$ are expected to differ in $2 p(1-p) n$ bits. Similarly, a random binary string of length $n$ is expected to differ in $p n$ bits from the centroid. While this behavior is only in expectation, when $n$ is large we can achieve it with high probability. Define distance as the Hamming distance normalized by the length $n$. Given a set of randomly generated binary strings, let $D$ be the minimum distance among all strings, and $d$ the maximum distance of any string to the centroid. If the set of strings is reasonably small and the length of strings is long, standard probability theory tells us that, with high probability, $D$ will be close to $2 p(1-p)$ and $d$ will be close to $p$, resulting in $D=2 d(1-d)$. We conjecture that this curve imposes a theoretical bound when given a large enough set of strings. To create a population of maximally separated (a large $D$ ) strings with minimal distances (a small $d$ ) to the centroid, one would seek to maximize the ratio $D / d$ for a given value of $p$. Here, $d$, which approaches the frequency of derived states $p$, is a measure of sequence divergence from the centroid. Thus, the maximized $D / d$ represents the maximal possible sequence divergence among the evolved strings at a given level of evolutionary divergence. Without constraining on $d$ (as in a separate algorithm shown below), the strings would diverge without regard to any biologically realistic constraints including the time since the evolutionary origin and functional and structural conservation. We used a genetic algorithm to validate the theoretical boundary with $n=100$ bits and $N=10$ strings. Distances optimized with the genetic algorithm (GA package in R [67]) were compared with empirical results based on the natural $\mathrm{OspC}$ variants as well as with results of randomly generated binary strings without $D / d$ maximization.

In a separate experiment, we used a genetic algorithm to search for a sample of maximally separated antigen variants to represent a MAD population, without consideration of a centroid. The searching was performed using the GA package in $R$ [67] to maximize $D$ (as defined above)fit $=\min \left(d_{i, j}\right)$. We then searched for centroid variants, using a separate genetic algorithm to minimize $d=\max \left(d_{i, j=1: 10}\right)$ fit $=\max \left(d_{i, j=1: 10}\right)$, where $i$ is an artificial centroid variant and $j$ is one of the ten simulated antigen variants. A centroid allele is chosen to minimize $d$. Evolutionary analogs were validated with a neighborjoining tree based on pairwise Hamming distances. An R markdown of the simulation protocol is included as Supplementary Information Text S2.

We note the similarity between the simulated maximally diversified antigens and well-separated binary codewords under the Hamming distance. We further note that the problem of finding centroids given a set of strings is known as the Closest String or the Hamming Centroid problem in computer science. While coding theory provides various techniques for generating well-separated codewords, and many algorithms for finding centroids exist [68, 69], we approached both problems in one stochastic framework based on genetic algorithms as described above.

\section{RESULTS}

Lack of immune cross-protection among $B$. burgdorferi strains in nature

In a previous study, B. burgdorferi strain diversity was quantified with high-throughput sequencing of the $\operatorname{ospC}$ locus at the level of single $I$. scapularis ticks [54]. Consistent with earlier results based on DNA cloning and DNA-DNA hybridization, the results reaffirmed a largely uniform distribution of a diverse set of $B$. burgdorferi strains identifiable by $\sim 16$ major-group ospC alleles in the highly endemic regions of Lyme disease in the Northeast US $[22,42]$. Using the same data set (Supplementary Information Data S1), we tested if frequencies of pairs of B. burgdorferi strains co-infecting a single tick were higher, lower, or equivalent relative to the expectation of random allelic association. With a sample of $n=119$ infected $l$. scapularis ticks, we found that the majority of strain pairs were overrepresented relative to random expectations and no pair was significantly underrepresented in infected ticks (Fig. 2). In particular, strain pairs containing the OspC variants $\mathrm{F}$ and $\mathrm{J}$ were the most overrepresented. To account for the possible contribution of the multiple blood meals of the adult ticks to the observed overrepresentation of mixed infections in single ticks, we repeated the permutation test using only the 25 nymphal ticks. All strain pairs were 


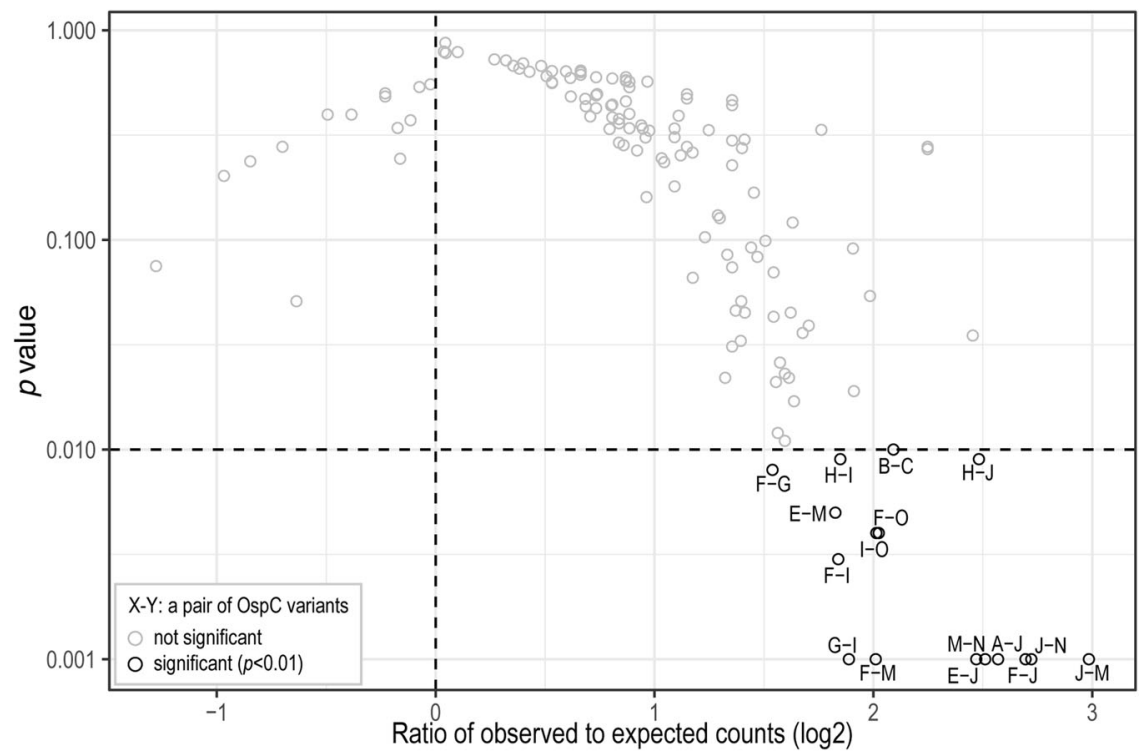

Fig. 2 Overrepresentation of multiple B. burgdorferi strains infecting single ticks. Each point represents a pair of OspC variants detected in a total of 119 infected I. scapularis ticks by deep sequencing [23]. The co-occurrence of an OspC pair was quantified by the ratio of the observed to the expected counts ( $x$-axis; log2 scale) and by the statistical significance ( $y$-axis; $p$ value, log 10 scale). Most OspC allelic pairs were more abundant than expected by chance (i.e., skewed to the right with log 2 ratio $>0$ ).

overrepresented, eight of which significantly so (results not shown). Since the $B$. burgdorferi strain diversity in individual ticks are driven mainly by the pathogen diversity in the host, the predominance of overrepresentation of mixed strain pairs supported immunological distinctness of OspC variants and a lack of immunological cross-protection against superinfection of the reservoir hosts $[22,23,53]$.

\section{ELISA testing of antigenic specificity of OspC variants with variant-specific sera}

In a previous study performed in one of our labs, $\mathrm{C} 3 \mathrm{H}$ mice were immunized with the 16 recombinant OspC natural variants [47]. These variant-specific sera were used to test the cross-reactivity of the OspC variants by ELISA. The authors identified five OspC variants $(B, E, F, I$, and $K)$ that were most broadly reactive with the variant-specific sera, consistent with results using naturally infected sera from human patients, dogs, and $P$. leucopus mice [47]. Here we re-analyzed the ELISA data set (Supplementary Information Data S2) by correcting for serum-to-serum variation with the use of normalized $z$-scores (Fig. 3 ). The $\mathrm{OD}_{450}$ readings showed stronger reactivity of homologous than heterologous bindings but large serum-specific variability (Fig. 3A). Some variant-specific sera (e.g., sK, for K-specific serum) showed consistently higher readings than others, reflecting experimentspecific factors such as a strong immune response of an animal. Without normalization to remove such experiment-specific background noise, the antigenicity of purified recombinant OspC displayed large variability and thus lacked statistical power for comparison (Fig. 3B). Indeed, the raw readings of homologous bindings were generally lower than the readings of heterologous bindings for the OspC variants. Normalization with respect to sera removed serum-specific background without altering the reactivity rankings or variance for each variant-specific serum (Fig. 3C). Critically, normalization restored the expected stronger reactivity of homologous than heterologous bindings while greatly reducing the reactivity variance for individual OspC variants (Fig. 3D). Thus, antigenicity of OspC variants could be compared with greater statistical confidence, such as the top cross-reactivity of $r E$ (for recombinant $E$ variant), $r K, r l, r F$, and $r B$ variants, in increasing order of cross-reactivity with a median value of $z>0$.
Normalization did not nullify but increased statistical confidence of the conclusion of the original study, which identified the same set of variants among the top cross-reactive variants [48].

The serum-normalized ELISA readings showed that, with two exceptions, rOspCs reacted significantly (i.e., with $z \sim 2.0$, two standard deviations above the mean) with homologous sera, indicating high antigenic specificity of rOspCs (Fig. 4, bar plot). The two exceptions included the variant $F$, which reacted significantly with both the F- and the B-specific sera, and the variant $\mathrm{J}$, which reacted more strongly with the $\mathrm{M}$-specific serum than with the J-specific serum. The high antigenic specificity of rOspCs is alternatively visualized with a heat map, which shows a strong diagonal line indicating the highest reactivity of rOspCs with homologous sera (Fig. 4, heat map). Note the absence of reactivity with the L-specific serum in both the bar graph and the heat map. This is because the strain expressing the $L$ variant was not available for generating the L-specific serum at the time [48]. Note also that although heterologous bindings were generally weaker than homologous bindings, rOspCs nonetheless reacted with heterologous sera. Notice that a binding value of $z=0$ represented the average reactivity, not an absence of antigen-serum binding.

An antigen reaction characteristic (ARC) curve was a way to summarize the overall reactivity of a $\mathrm{rOspC}$ with all serum samples (Fig. 4, ARC curves). In addition, the ARC curves provided a quantitative measure of antigen specificity and cross-reactivity, showing the most broadly reactive antigens at the top and the most specific antigens at the bottom. For example, the ARC curves show $B, F, K, E$, and I being the most broadly reactive variants, in agreement with the conclusion of the original study [47].

\section{Immunoblot assays with variant-specific sera}

We further tested the antigenic specificity of rOspCs with the use of immunoblot assays and a full set of 16 variant-specific sera (the $\mathrm{L}$-specific sera included) from immunized $\mathrm{C} 3 \mathrm{H}$ and $P$. leucopus mice (Fig. 5, top). The raw immunoblot images showed strong specific reactions of $\mathrm{rOspC}$ with homologous sera (diagonal) and weak reactions with heterologous sera (off-diagonal). As in the ELISA analysis, we corrected for serum-to-serum variation by normalizing binding intensities with respect to sera (Supplementary Information Data S3). Consistent with ELISA results, the re-scaled intensities 

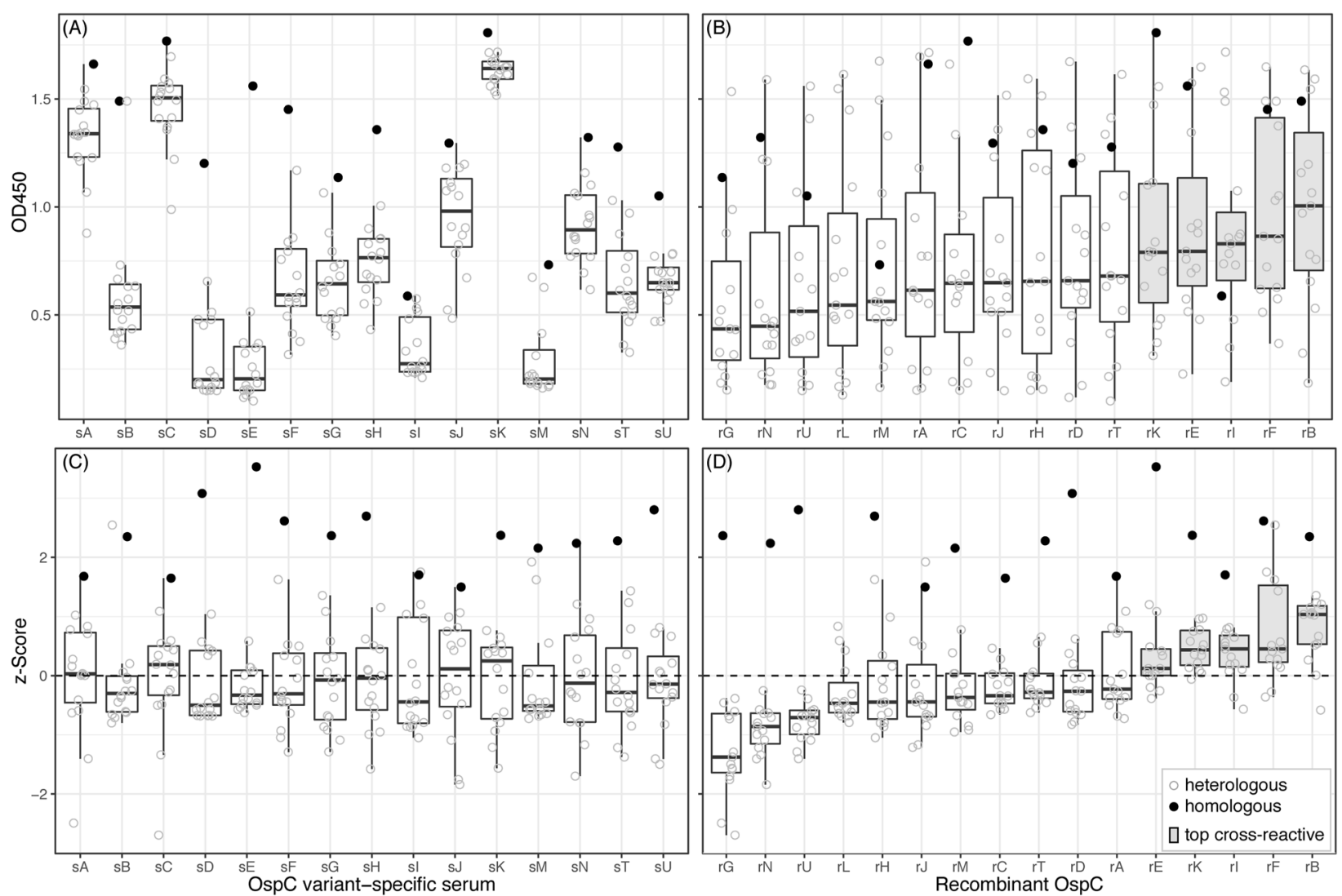

Fig. 3 Removal of serum-specific background noise with normalization. A Raw $\mathrm{OD}_{450}$ readings ( $y$-axis) from a previously published ELISA of the binding between 16 purified recombinant OspC antigens with 15 OspC variant-specific mouse sera ( $x$-axis) [48]. Homologous bindings (solid dots) were between an antigen variant and a serum from a $\mathrm{C} 3 \mathrm{H}$ mouse immunized with the same recombinant variant. Heterologous bindings (open dots) were between an antigen variant and a serum from a mouse immunized with a different variant. ELISA readings varied significantly among the sera $(F=51.46, p<2.2 \mathrm{e}-16$, by an ANOVA). B OD 450 readings with respect to the 15 recombinant OspC variants $(x-$ axis), ordered by the medians. Without correcting for the serum-by-serum variability, the OspC variants did not vary significantly in reactivity with the variant-specific sera $(F=1.04, p=0.42)$. C Normalized reactivity ( $z$-score, $y$-axis) with respect to the variant-specific sera $(x$-axis). Serum-specific variability was removed $(F=0, p=1)$. D Normalized reactivity ( $z$-score, $y$-axis) with respect to the OspC variants $(x$-axis). After normalization, the OspC variants showed significant variability in reactivity with the variant-specific sera $(F=6.17, p=8.3 \mathrm{e}-11)$. Five OspC variants with a median $z>0$, indicating above-average reactivity, were highlighted with shaded boxes. The same five OspC variants ranked as the most reactivity without normalization. Thus, normalization did not change the ranking but greatly improved statistical confidence and precision for comparing the antigenicity among the antigen variants.

showed the strongest bindings between rOspCs with homologous sera (Fig. 5, heat map). However, the ARC curves showed a lack of consistency in the topmost cross-reactive rOspC variants between the immunoblot using the $\mathrm{C} 3 \mathrm{H}$ mice ( $\mathrm{rH}$ and $\mathrm{rl}$ at the top) and the immunoblot using the $P$. leucopus mice ( $r T$ and $r$ J at the top) (Fig. 5, ARC curves). Further, the rOspC rankings of the immunological breadth as quantified by the ARC curves in both immunoblots were different from the ranking from the ELISA experiment using the $\mathrm{C} 3 \mathrm{H}$ mice ( $r B$ and $r F$ at the top, Fig. 4, ARC curves).

To conclude, testing on the basis of ELISA and immunoblots and with the use of OspC variant-specific sera from two mouse species all showed the strongest reactions of OspC variants with homologous sera. Reactions of OspC variants with heterologous sera, however, were weaker and inconsistent between experiments and between the two mouse species.

\section{Centroids reacted broadly with naturally infected human and mouse sera}

We designed six evolutionary analogs (Supplementary Information Text S1) expected to show broad antigenic cross-reactivity with the 16 natural OspC variants with the use of three evolution-based algorithms (Fig. 1). The root analog ("Root") is defined as the maximum-likelihood sequence of the hypothetic phylogenetic root of the 16 natural OspC variants.
The consensus sequence ("Consense") consisted of majority amino-acid residues at individual alignment positions. The centroid analogs ("Centroid") were computationally derived sequences that were minimized for sequence differences with the 16 natural variants. Whereas the root and consensus algorithms each generated a single OspC analog, the centroid algorithm generated ten optimized sequences from each run. To increase the diversity of candidate sequences, the algorithm was ran with repetition and the most optimized sequence was chosen form each run. We chose four centroids among a dozen candidates for further experimentation on the basis of their distinct phylogenetic positions.

Effectively, the algorithms drastically cut the sequence differences of the evolutionary analogs with the natural OspC variants (e.g., $d=0.182 \pm 0.024$ for the consense) to approximately half of the sequence differences among the natural variants themselves $(d=0.260 \pm 0.033)$. Based on immunological models suggesting a tight correlation between sequence and antigenic distances [70], we expected a similar level of reduction in the antigenic distances of each evolutionary analog to the natural OspC variants. Phylogenetic analysis of these evolutionary analogs validated their expected central positions among the OspC sequence diversity (Fig. 6C). Sequence differences of the evolutionary centroids with the 16 natural variants were more 

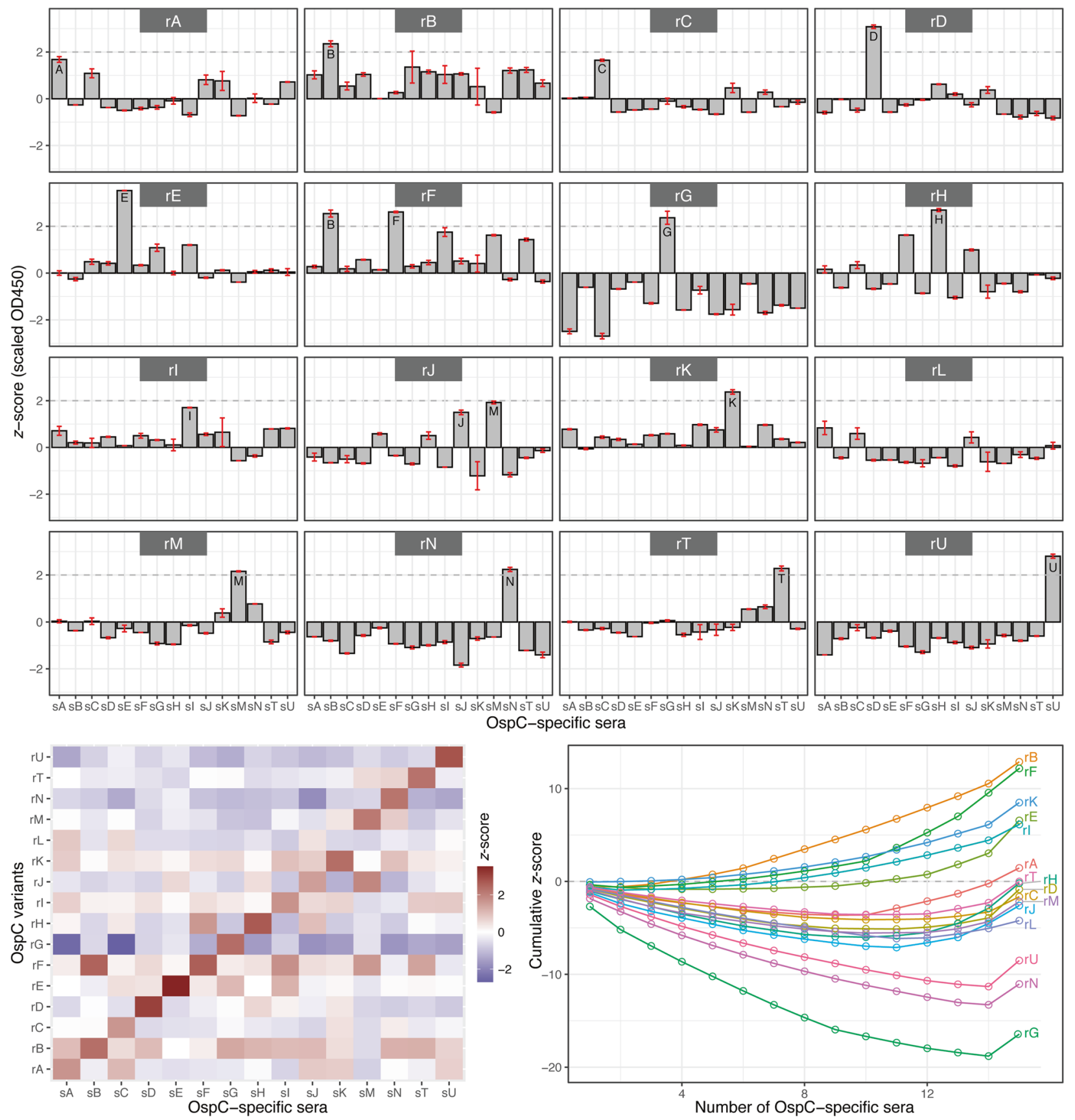

Fig. 4 ELISA of OspC variants with variant-specific sera. Fifteen sera ("sA" through "sU") from mice, each immunized with a specific recombinant OspC variant, were previously assayed for reactivity with the 16 OspC variants ("rA" through "rU") using ELISA [48]. (Top) Each panel shows binding intensities (normalized $z$-scores, $y$-axis) of an OspC variant with a panel of OspC-specific sera ( $x$-axis). Error bars show one standard deviation above and below the mean from two replicated assays. A value above the $z=2$ line (dashes) indicates a highly significant reaction. (Bottom left) A heat map representation of the mean z-scores. (Bottom right) Antigen reaction characteristics (ARC) curves, similar to the receiver-operation characteristics (ROC) curves, is a measure of antigen specificity. Each curve traced the cumulative $z$-scores ( $y$-axis) of an OspC variant's binding intensities with the sera samples, ordered by the lowest to the highest reactivity. The ARC curve rises with an aboveaverage binding value $(z>0)$ and drops with a below-average binding value $(z<0)$. Thus, a high-rising curve (e.g., for $\mathrm{rB})$ indicated consistently above-average reactivity with sera samples, suggesting a broadly cross-reactive antigen. Conversely, a low-lying curve (e.g., for rG) indicated consistently below-average reactivity, suggesting a relatively specific antigen. Curves close to the zero line (the majority of variants) indicated antigens with an average level of cross-reactivity.

uniform while the consensus analog showed a lower average difference (Fig. 6A, B; Supplementary Information Data S4). The root analog showed the highest average sequence difference as well as the highest variability in sequence differences with the natural variants (Fig. 6B).
We cloned, overexpressed, and purified the six evolutionary analogs and the 16 natural variants as recombinant proteins (Supplementary Information Fig. S1). Antigenicity of each rOspC was quantified by its reactions with OspC-positive sera (Table 1) from naturally infected human patients $(n=41)$ and $P$. leucopus 

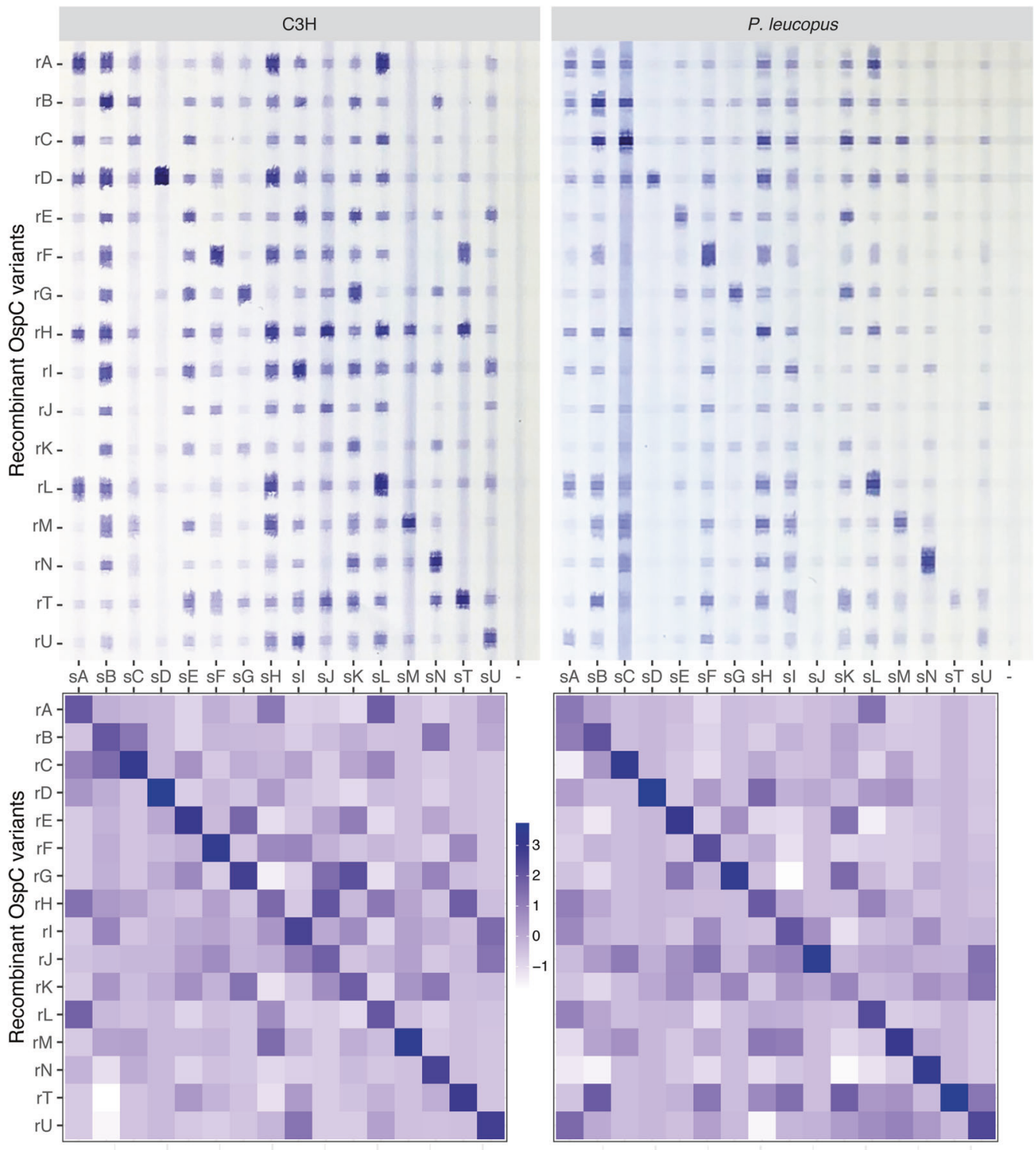

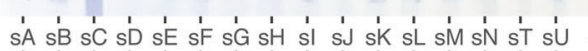
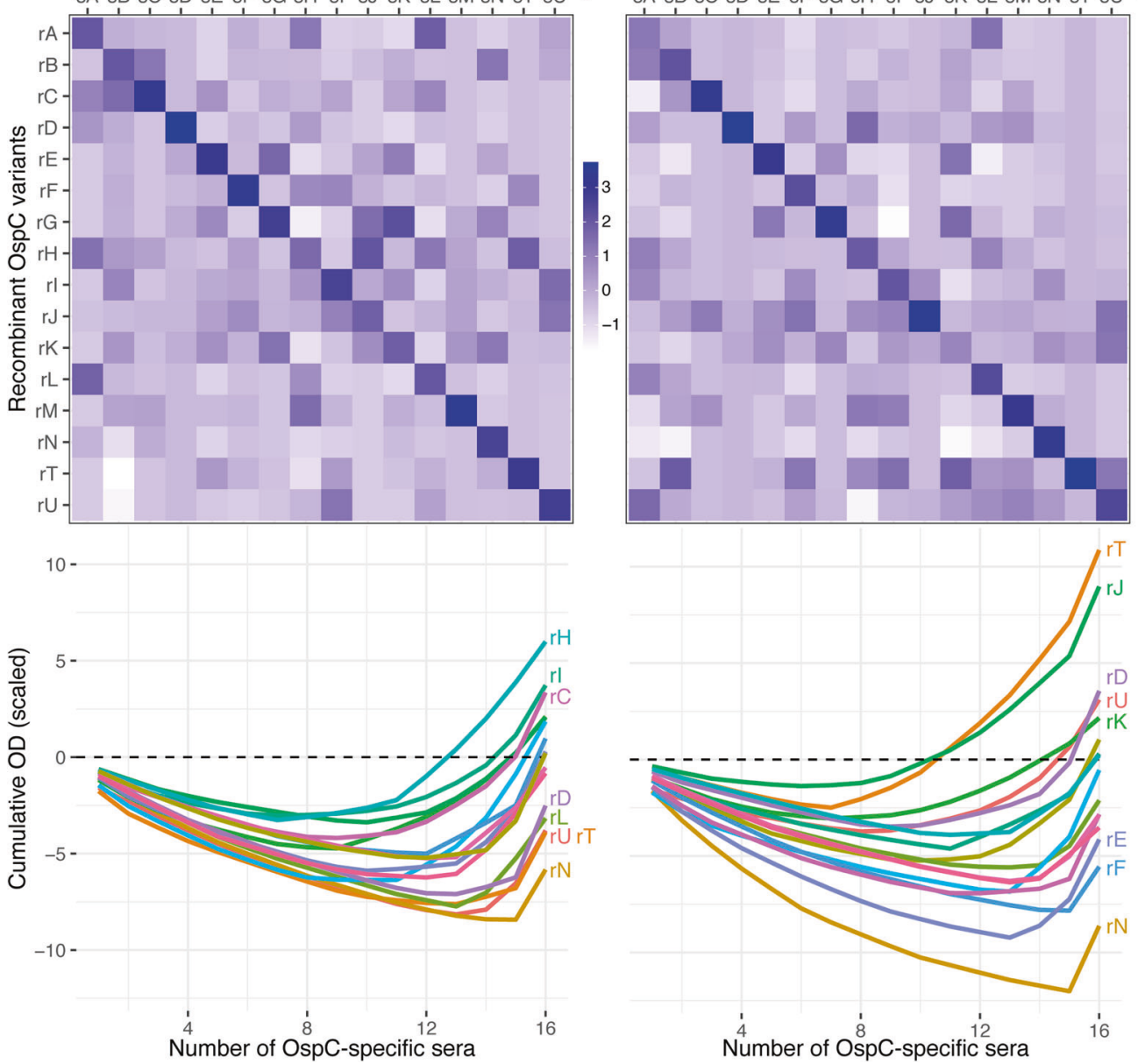

Fig. 5 Immunoblot testing of OspC variants with variant-specific sera. (Top) Immunoblot images of OspC-specific sera ( $x$-axis) from the C3H mice (left) and the $P$. leucopus mice (right) reacting with recombinant OspC variants ( $y$-axis). The last column (labeled with "-") is the negative control, showing reactions of sera from un-immunized mice. (Middle) Corresponding heatmaps. The binding intensity values on the immunoblot images were captured by ImageJ [64]. Values were then normalized by subtracting intensities from the negative controls and by scaling to $z$-scores. (Bottom) ARC curves. Some of the most (topmost) and the least (bottom-most) reactive recombinant OspC variants were labeled. 

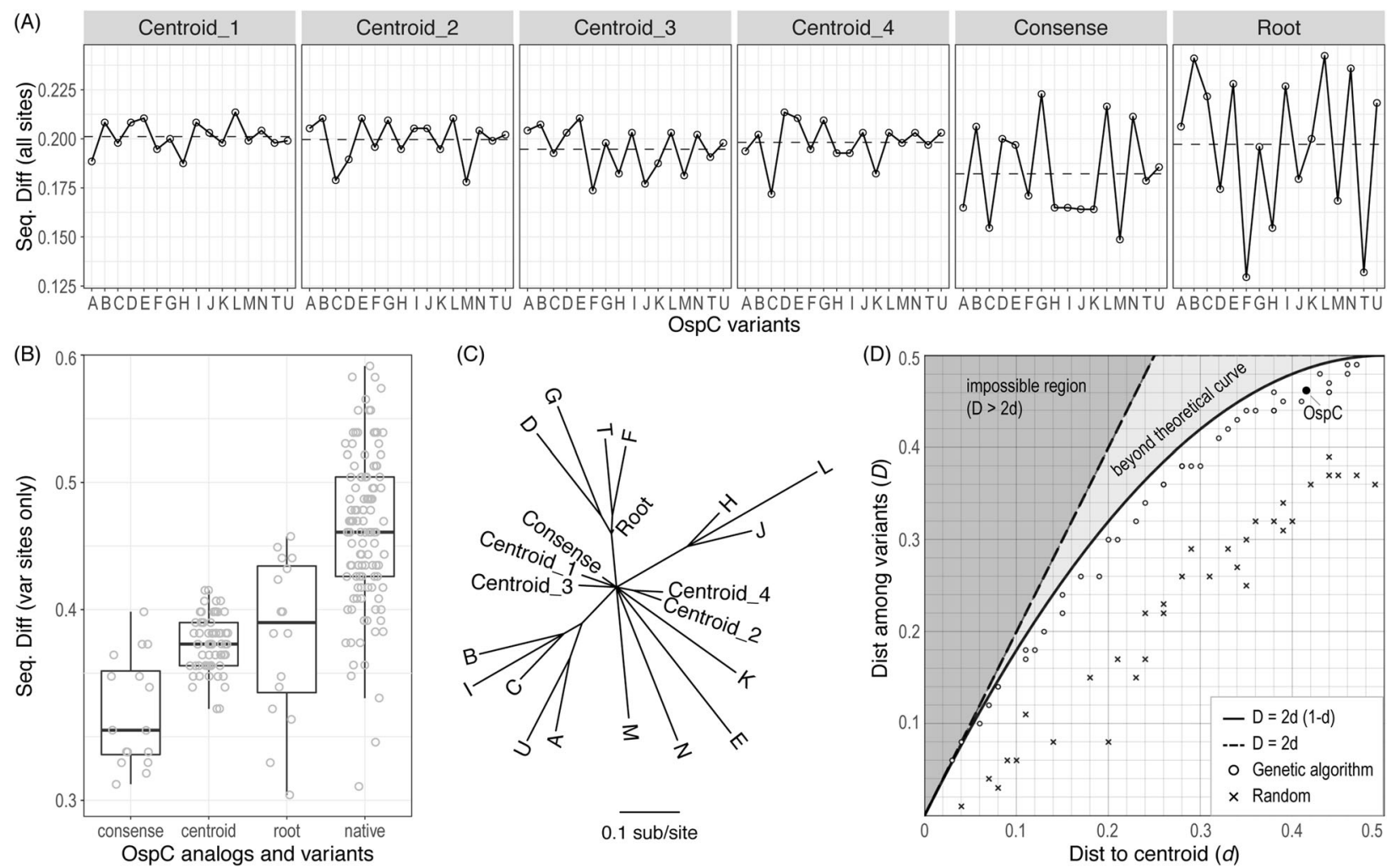

(C)

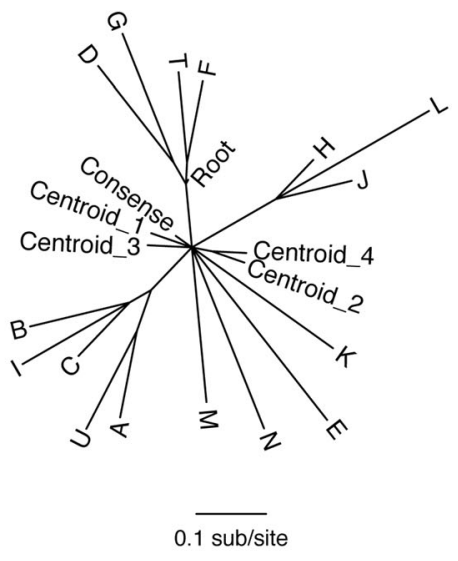

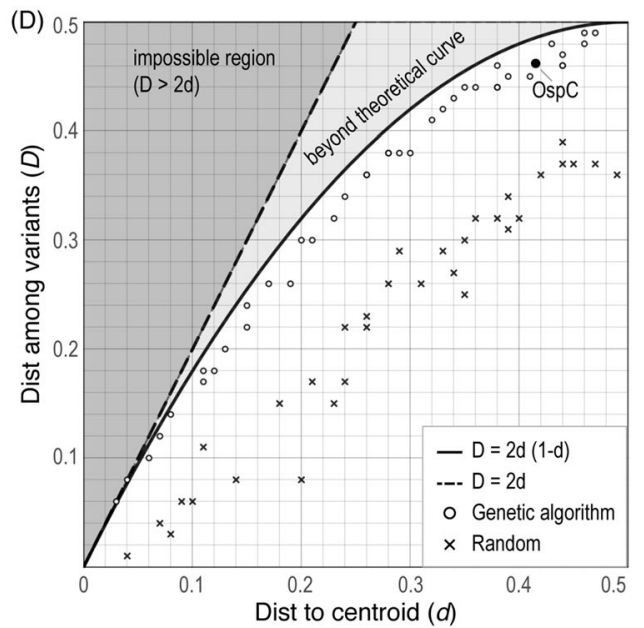

Fig. 6 Sequence differences and the binary model. A The $y$-axis shows the fraction of amino-acid differences out of the total number of aligned un-gapped residues (ranging from 190 to 196 amino acids) between an evolutionary OspC analog and a natural OspC variant. The 16 natural $B$. burgdorferi OspC variants were listed alphabetically on the $x$-axis. The dashed lines indicate the averages. B Boxplot shows the fraction of sequence differences out of the total number of variable sites (115 alignment sites, gaps included) for the evolutionary analogs to the 16 natural variants ("consense", "centroid", and "root"), as well as the sequence differences between pairs of the natural variants themselves ("native"). Solid lines in the middle of the boxes represent the medians. C A maximum-likelihood tree of the 16 natural OspC variants common in the Northeast US and the six evolutionary analogs, including the mid-point root sequence ("Root"), a consensus sequence ("Consense"), and four centroids ("Centroid"). All branches are supported by a bootstrap value of 0.8 or above. D A binary model of antigen divergence. A genetic algorithm was used to find 10 binary strings of length 100 and their centroid with a goal to maximize $D / d$, where $D$ and $d$ are the distances among the 10 strings and from the strings to the centroid, respectively (see Methods). Observe that $D / d$ cannot exceed 2 by triangular inequality. The strings are generated with a probability of " 1 " equal to $p \leq 0.5$ and their centroid is initialized to a string of 0 s. The values of $D$ and $d$ from populations of randomly generated strings ("Random") for various values of $p$ in $[0,0.5]$ are shown. Runs of the genetic algorithm on these strings show that the resulting $(d, D)$ matched very well the boundary of the theoretical curve given by $D=2 d(1-d)$ (see Methods). The corresponding point $(D=0.462$ and $d=0.415)$ for OspC is also shown, suggesting that the OspC variants are undergoing a similar diversifying selection towards maximum sequence separation. Note that the distance $D$ for OspC was obtained as the average distance rather than the minimum distance due to the presence of sequences that were too close in the phylogeny (e.g., a difference of 0.281 between the $\mathrm{H}$ and $\mathrm{J}$ variants), which artificially lowers the minimum distance.

mice $(n=10)$ using ELISA (Supplementary Information Data S5). Natural OspC variants (gray bars) reacted with the human serum samples with visible variability, so did the root (orange bars) and the consensus (blue bars) analogs (Supplementary Information Fig. S2). One centroid ("CT1") reacted poorly with the majority of mice sera (Supplementary Information Fig. S3). In contrast, the other three centroids ("CT2", "CT3", and "CT4") reacted consistently at high levels with all sera. The mouse sera reacted with rOspCs in a more variant-specific manner than the human sera. For example, the mouse sera P03, P04, P06, P08, and P09 reacted strongly with one to four natural rOspC variants while weakly with other natural variants (Supplementary Information Fig. S3). Although the natural rOspC variants reacted strongly with some of the murine sera, the three centroids reacted consistently high with all murine sera.

The antigenic breadths of the OspC variants were further quantified with the use of heat map (Supplementary Information Fig. S4) and the normalized $z$-scores (Fig. 7). In the heat map, the OD450 readings were scaled with respect to individual sera and, subsequently, both the sera (in columns) and the rOspCs (in rows) were grouped according to pairwise similarities in reactivity (Supplementary Information Fig. S4). The three centroids (CT2, CT3, and (T4) showed as a distinct cluster that reacted with the human and mouse sera at levels that were consistently above the average.

The boxplots of the serum-normalized $z$-scores confirmed significantly broader reactivity of all evolutionary analogs relative to the natural variants with the naturally infected $P$. leucopus sera, with $p=0.031$ for the root analog, $p=4.9 \mathrm{e}-03$ for the consense analog, $p=0.041$ for CT1, $p=5.2 \mathrm{e}-05$ for $\mathrm{CT} 2, p=1.4 \mathrm{e}-06$ for CT3, and $p=5.6 \mathrm{e}-05$ for CT4 (Fig. 7 top left, boxplot). With the use of naturally infected human sera, reactivity of CT2 $(p=9.1 \mathrm{e}$ $-10)$, CT3 $(p=2.4 \mathrm{e}-06)$, and CT4 $(p=1.1 \mathrm{e}-09)$ was significantly higher than the natural variants. The reactivity of CT1 $(p=2.3 \mathrm{e}$ -06) was significantly lower than the antigenicity of the natural variants while the reactivity of the root $(p=0.31)$ and consense $(p=0.065)$ analogs was not significant (Fig. 7 bottom left, boxplot). The ARC curves summarized the strong reactions of the three evolutionary centroids with the sera (the top three curves) and the weak reaction of the root and consensus analogs (Fig. 7 top and bottom right, ARC curves). The ARC curves of the cross-reactivity of natural OspC variants with the human sera (gray lines) showed $\mathrm{rB}$ as a top cross-reactive and $\mathrm{rG}$ as the least cross-reactive variant, consistent with the rankings of these two 

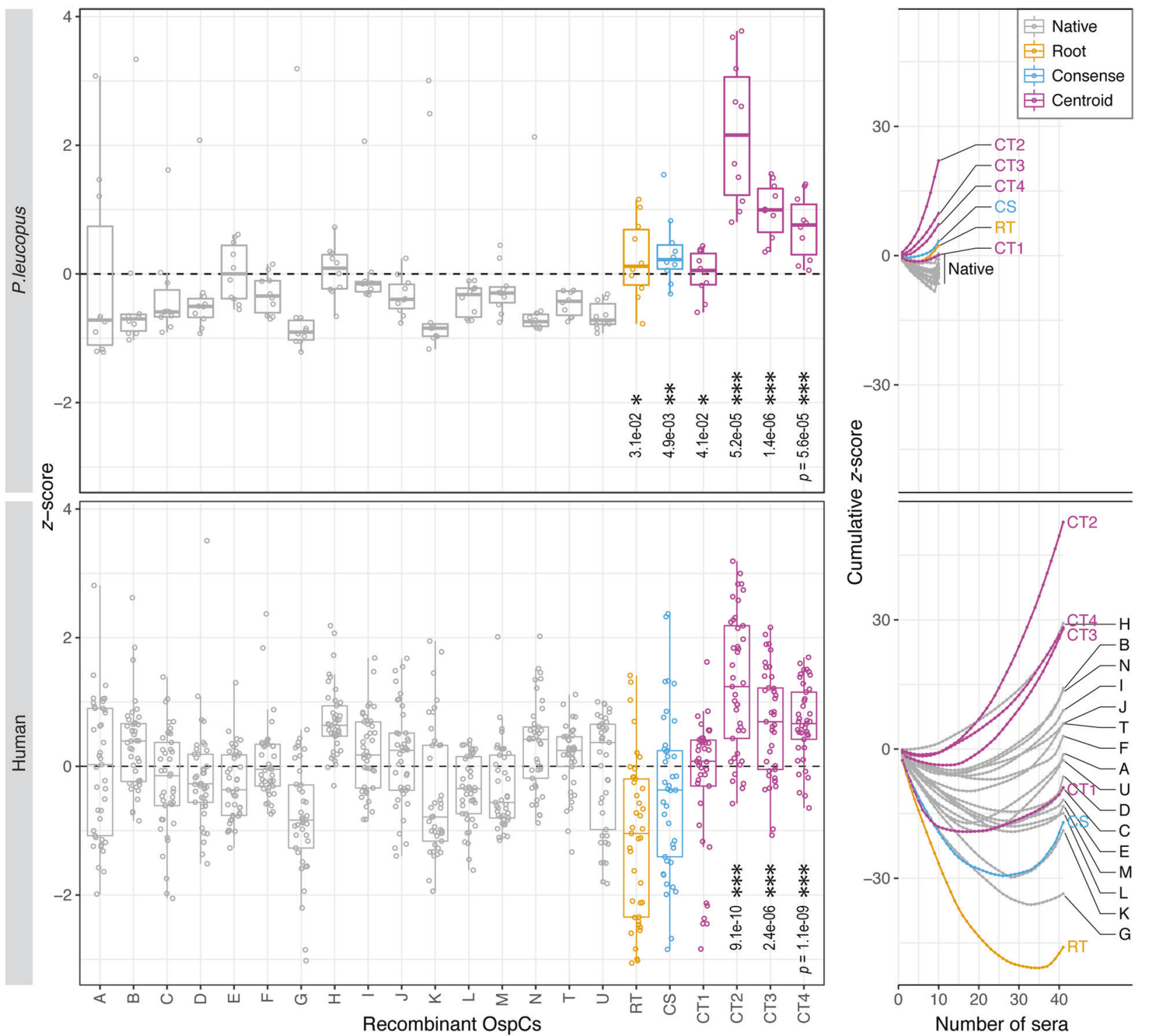

Fig. 7 Reactivity of synthetic analogs with naturally infected human and mouse sera. (Top left) Reactivity ( $z$-score, $y$-axis) of natural OspC variants $(n=16)$ and evolutionary analogs $(n=6)$ ( $x$-axis) with sera from naturally infected P. leucopus mice $(n=10)$. All six evolutionary analogs showed significantly higher (with $t$ tests) reactivity with the sera of the reservoir host species than the reactivity of the natural variants as a group. (Top right) Antigen reaction characteristics (ARC) curves showed consistently high reactivity of the six evolutionary analogs, indicating their broader antigenicity relative to the natural OspC variants in reacting with the sera of the reservoir hosts of $B$. burgdorferi. (Bottom left and right) Corresponding graphs with sera from naturally infected human patients $(n=41)$. Three centroids (CT2, CT3, and CT4) showed significantly higher (with $t$ tests) reactivity than the reactivity of the natural variants as a group. Reactivity of the other three evolutionary analogs (CT1, Consense, and Root) was significantly lower than or not significantly different from the reactivity of the natural antigen variants.

variants in the ARC curve based on ELISA with variant-specific sera (Fig. 4 bottom right, ARC curves). We conclude from the ELISA testing that the evolutionary centroids with computationally optimized sequence differences tended to react more broadly with naturally infected human and mouse sera than natural OspC variants.

\section{Evolutionary analogs are structurally similar to native OspC variants}

The CT1 centroid was more cross-reactive than the natural variants with the mouse sera but, unlike the CT2, CT3, and CT4, less crossreactive than many natural variants with the human sera (Fig. 7, top and bottom right, ARC curves). We had expected CT1 to be the most cross-reactive among the four centroids because it had the lowest variance in sequence differences to the natural variants (Fig. $6 \mathrm{~A}$ ). It appeared that low sequence differences with the natural variants were an essential but not sufficient predictor of broad OspC crossreactivity. To investigate structural factors contributing to the antigenic breadth of OspC variants, we obtained a structural alignment of the evolutionary analogs with a solved OspC structure (PDB ID: 1F1M) [71] (Supplementary Information Fig. S5). Measurements of structural variability showed the high structural similarity of the evolutionary analogs with the natural OspC variants as well as among the evolutionary analogs themselves (Supplementary Information Data S6). The structural alignment provided a basis for further comparative analysis to identify the amino-acid residues associated with the variability in antigenicity among the natural and synthetic OspC variants. 


\section{DISCUSSION}

\section{High antigenic specificities of natural OspC variants}

Previous field-based studies have established an overabundance of ticks infected by a mixture of Lyme pathogen strains identified by their ospC alleles $[23,45]$. In the present study, we further tested immunological distinctness of diverse $B$. burgdorferi strains co-existing in the Northeast US using field-collected I. scapularis ticks. Composition of $B$. burgdorferi strains in individual infected ticks especially in nymphs-having fed on a single blood meal from a single host-faithfully reflects the spirochete composition in reservoir hosts $[23,53]$. As such, we expected that the frequency of mixed infection by a pair of strains to be lower than expected by chance if the cross-protection of reservoir hosts against superinfection by multiple strains was common in nature. The present statistical analysis of coinfection rates reaffirmed an overabundance of pairs of $B$. burgdorferi strains carrying distinct OspC variants (Fig. 2). In conclusion, reservoir hosts of Lyme pathogens tend to be infected by multiple strains, indicating a lack of cross-protective immunity in reservoir hosts. By extension, we conclude the immunological distinctness of $B$. burgdorferi strains carrying different $0 s p C$ alleles in nature.

Experimental infection in laboratories using $B$. afzelii, a Lyme pathogen common in Europe and Asia, showed that mice immunized with one recombinant $\mathrm{OspC}$ variant protected the host from infection by a strain carrying the homologous OspC variant but not by the strain carrying a heterologous OspC variant [37]. These strains, however, differed in genomic background besides the ospC sequences. Immunological mechanisms by which the host serum neutralizes spirochetes carrying a homologous but not a heterologous ospC allele was elucidated using genetic manipulations and immunodeficient mice, firmly establishing the causal role of the OspC molecule in eliciting strainspecific protective humoral immunity in B. burgdorferi hosts [39]. Sequences that are conserved among OspC variants, e.g., the $C 7$ and C10 domains, are unlikely to be the targets of NFDS and indeed do not elicit protective immunity [46, 49]. Instead, immunodominant epitopes have been mapped to the highly variable regions including the C-terminus domains [47, 72]. Furthermore, conformational epitopes and structural integrity of the $\mathrm{OspC}$ molecules are required to trigger protective immunity $[60,73,74]$.

By immunizing the $\mathrm{C} 3 \mathrm{H}$ mice and the reservoir species $P$. leucopus with recombinant $\mathrm{OspC}$ proteins and quantifying antigenic reactions using ELISA and immunoblots, a previous study [48] and the present work demonstrated the high antigenic specificities of natural OspC variants with the homologous sera, and the much diminished reactivity of OspC variants with the heterologous sera (Figs. 4, 5).

To summarize these field-based and lab-based studies, we use the term MAD to describe the immunological distinction of natural OspC variants and their dominant role in maintaining $B$. burgdorferi diversity in nature. Evidence of antigenic separation among natural OspC variants emerged first from population genetic surveys of $\operatorname{osp} C$ sequence variability and allele frequencies in natural B. burgdorferi populations, which showed strong balancing selection driving genetic diversity at the ospC locus mediated by ecological mechanisms including immune escape, host species specialization, or both $[22,25,42]$. Subsequent wholegenome sequencing revealed frequent recombination among coexisting strains and $0 s p C$ being a recombination hotspot as well as the most polymorphic single-copy gene in the $B$. burgdorferi genome $[40,75]$. We showed by forward-evolution simulation that the combined forces of homologous recombination and negativefrequency-dependent selection were sufficient to explain the seemly paradoxical pattern of the high recombination rate at $\operatorname{ssc} C$ and the sequence hyper-variability at the same locus [40].

An epidemiological model offers a more intuitive understanding of the paradox of sustained linkage disequilibrium in the presence of genetic recombination at an antigen locus [3]. Using a token antigen consisting of two bi-allelic epitope sites (e.g., A1 and $A 2$ at site A, B1 and B2 at site B), Gupta et al. predicted complete linkage disequilibrium resulting in a population consisting of only $\mathrm{A} 1 \mathrm{~B} 1$ and $\mathrm{A} 2 \mathrm{~B} 2$ haplotypes without the crossover A1B2 and $A 2 B 1$ haplotypes, if it could be assumed that the host antibodies neutralize $A 1$ and $A 2$ (as well as B1 and B2) specifically without cross-reactivity (i.e., anti-A1 not binding $A 2$ and vice versa). This is because, in such a system the A1B1-genotyped microbes would survive the host producing antibodies against $A 2$ and $B 2$, the $A 2 B 2$-genotyped microbes would survive the host producing antibodies against $\mathrm{A} 1$ and $\mathrm{B} 1$, but the $\mathrm{A} 1 \mathrm{~B} 2-$ or $\mathrm{A} 2 \mathrm{~B} 1$ genotyped microbes would not survive either host. This simple epidemiological model thus predicts maximum antigenic divergence (two bits of difference between $\mathrm{A} 1 \mathrm{~B} 1$ and $\mathrm{A} 2 \mathrm{~B} 2$ ) when the host immunity is highly allele-specific. This model, known as the strain theory, has been further refined and used to understand the stable coexistence and the temporal persistence of diverse strains in natural pathogen populations including the influenza $A(H 3 N 2)$ virus and malaria $[6,76]$.

\section{Mechanism of maximum antigen diversification: a mathematical model}

To explore immunological and molecular mechanisms underlying the broad antigenicity of evolutionary centroids, we proposed a mathematical model in which antigen sequences in a pathogen population were represented by binary strings of $1 \mathrm{~s}$ and $0 \mathrm{~s}$ (see Methods). Although there are potentially 20 amino-acid states at each alignment site, the binary representation of antigen sequences is justified on the basis of sequence variability within pathogen populations which consists predominantly of singlenucleotide changes. We subsequently simulated MAD and evolutionary centroids using genetic algorithms (simulation code available as Supplementary Information Text S2). Simulating OspC sequence evolution with genetic algorithm, which generates diverse binary strings through genetic mechanisms including random mutation and recombination, is justified on the basis that the ospC locus is a recombination hotspot on the circular plasmid cp26 in B. burgdorferi $[14,40,77]$. Note that in general genetic algorithms attain local maximal divergence but not a global maximum.

The binary model revealed a theoretical bound $D=2 d(1-d)$ at a particular evolutionary distance $p$, where $D$ is the minimum sequence distance among the binary strings, $d$ is the maximum distance of these strings to a centroid (represented by a string of $0 \mathrm{~s}$, i.e., all ancestral states, at the root), and $p$ is the probability of 1 (i.e., a derived state) (Fig. 6D). In this formulation, $p=d$, leading to $D=2 p(1-p)$. As the population evolves, $p$ increases and results in increasing sequence diversity among the strings $(D)$ as well as increasing distances to the centroid $(d)$, as shown by the random points in Fig. 6D. When the sequences are under selection for diversification from one another, the $D$ value deviates far above the random points and is maximized to $D=0.5$ at $p=0.5$. Indeed, the natural OspC variants are separated from one another at an average sequence difference of $D=0.462$ in fraction of variable sites (not counting the constant sites), in agreement with the maximum sequence separation driven by diversifying selection (Fig. 6B). Presence of recombination, more than mutation, is a key force driving the maximal and approximately uniform sequence divergence among the $\mathrm{OspC}$ variants. Simulated sequence divergence without recombination resulted in lower and more dispersed pairwise sequence differences (Supplementary Information Text S2).

Furthermore, the binary model indicated that the genetic algorithms we designed was effective in generating centroids close to the theoretical bounds (Fig. 6D). With a maximum distance of $d=0.415$, the binary model suggests that OspC centroids with smaller distances to the natural variants are unlikely 
Table 2. Simulated maximally diverged variants and evolutionary analogs $\mathrm{s}^{\mathrm{a}}$.

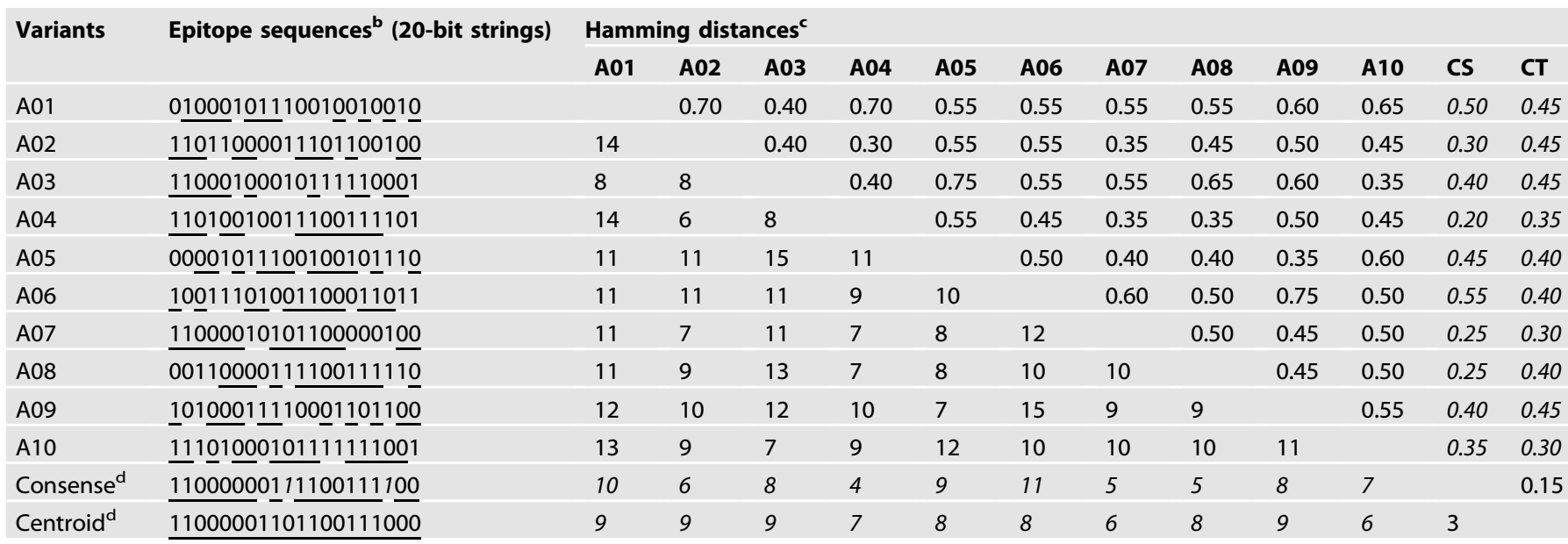

"Both the maximally divergent variants (A01 through A10) and the evolutionary analogs ("Consense"/"CS" and "Centroid"/"CT") were generated using genetic algorithms (analysis shown in Fig. 8; code shown in Supplementary Information S4 R Markdown).

${ }^{b}$ Each string represents an antigen consisting of 20 epitopes with two possible states ( 0 and 1$)$. Substrings identical to those in the Centroid are underlined to highlight the interleaved nature of antigen similarities.

'Hamming distances: pairwise string differences (lower triangle) and length-adjusted relative distances (upper triangle).

${ }^{d}$ Distances of the consense and centroid analogs are given in italic to indicate their relatively low distances to the simulated natural variants. Note that distances of the centroid are more uniform than those of the consense.

to be discovered given the length of variable site $(\sim 100$ amino acids) and this set of 16 natural variants. In summary, the binary model elucidates a theoretical boundary of OspC sequence variability within the $B$. burgdorferi populations as well as a theoretical limit in sequence distances of possible OspC centroids.

In a token model with the use of ten 20-bit strings, we simulated a population of maximally diverged antigens (Table 2) and validated the central positions of the consensus and centroid variants with a neighbor-joining tree based on pairwise Hamming distances (Fig. 8 top right, tree). Simulation results were further validated by tabulating pairwise Hamming distances into a distance matrix, which showed a narrow range of distances (6 to 9) for the centroid, and a wider range of distances (4 to 11) for the consensus, and large distances (6 to 15) between simulated natural variants (Table 2). We obtained $z$-scores by normalizing the sequence similarities with respect to individual simulated natural variants. As such, we were able to compare and show a high resemblance between the simulate results and experimental results using the immunized mice (Figs. 2, 3, 8). For example, both the simulated and experiment-derived results showed highly specific bindings $(z>2.0)$ between homologous variants (Figs. 4, 5, 8). Both the simulated and experiment-derived results showed the broad antigenicity of evolutionary analogs with heat map and ARC curves (Supplementary Information Fig. S4, Figs. 7, 8, corresponding heatmaps and ARC curves). Importantly, the simulated MAD population, mirroring experimental results, revealed that the broad antigenicity of evolutionary analogs was a result of consistently above-average $(z>0)$ reactivity even as their cross-reactions with any particular natural antigens remained uniformly lower than the strongest reactions between homologous reactions $(z<1)$ (Fig. 8 top left, bar plots).

However, the centroid algorithm did not succeed uniformly, suggesting that sequence similarities alone do not guarantee broad antigenicity. One of the centroids ("CT1") showed low reactivity with the human sera relative to the other three centroids despite being similar in the distribution of sequence distances and a lower variance. Structural integrity and finegrained epitope similarity must contribute to serum reactivity as well, despite an overall high structural similarity of the centroids with the native variants (Supplementary Information Fig. S5). Validation of structural similarity of evolutionary analogs to the natural OspC variants requires experimental interrogation with e.g., circular dichroism (CD) and nuclear magnetic resonance spectroscopy [46]. Computational and experimental structural analyses are needed to identify the structural determinants of variability in antigenicity among the OspC variants.

\section{Implications to diagnostic and vaccine development}

A new class of broad-spectrum diagnostics and vaccines could be designed by countering the evolutionary trend of maximum antigenic divergence in local $B$. burgdorferi populations. In diagnosis, the standard two-tiered testing (STTT) is based on EIA and immunoblots and lacks sensitivity for patients who develop acute erythema migrans, an early-stage Lyme disease $[62,78]$. The newly recommended modified two-tiered testing (MTTT) protocol consisted of two ElAs without immunoblot and improved the sensitivity of detecting early Lyme disease cases $[79,80]$. The use of multiple OspC variants may further improve diagnostic sensitivity with their broad reactivity with diverse $B$. burgdorferi strains [48]. With a similar ability to react with diverse $B$. burgdorferi strains and with a single antigen, the centroid antigens are novel diagnostic candidates if they pass specificity tests [61].

Currently there is no human-use vaccine against Lyme pathogens on the US market [81-83]. The design of currently available OspC/OspA-based vaccines for canine use was based on identification of immunodominant epitopes in individual OspC variants and concatenating them into linear multivalent superantigens $[46,72,84]$. A multivalent vaccine consisting of as many as eight OspC-type specific epitopes has been shown to be immunogenic [49]. Because of the large number of OspC variants co-circulating in a local endemic area (e.g., $\sim 20$ in the Northeast US), it is unclear the efficacy of chimeric vaccines to elicit broadly protective immunity in humans $[50,85]$. Critically, studies have shown that immune protection of OspC-based vaccines required the presence of native OspC structure including dimerization, suggesting that much of the neutralizing antibodies targeted structural rather than linear epitopes [73, 74]. 

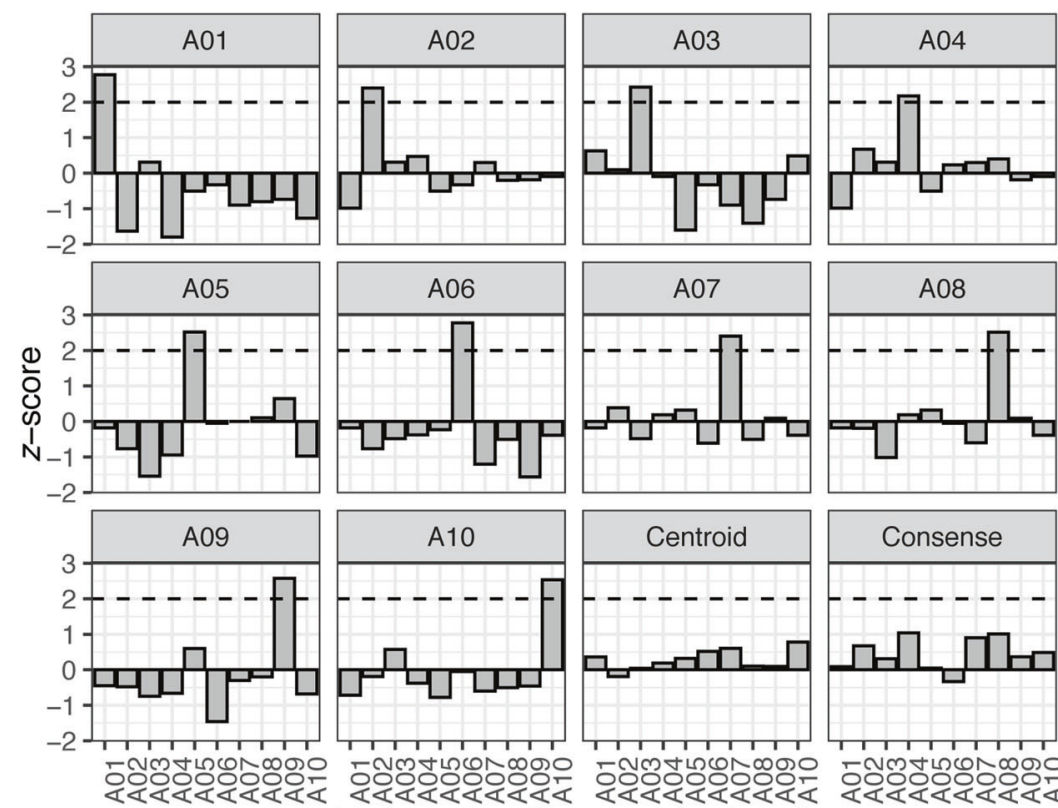

Variant-specific sera (simulated)
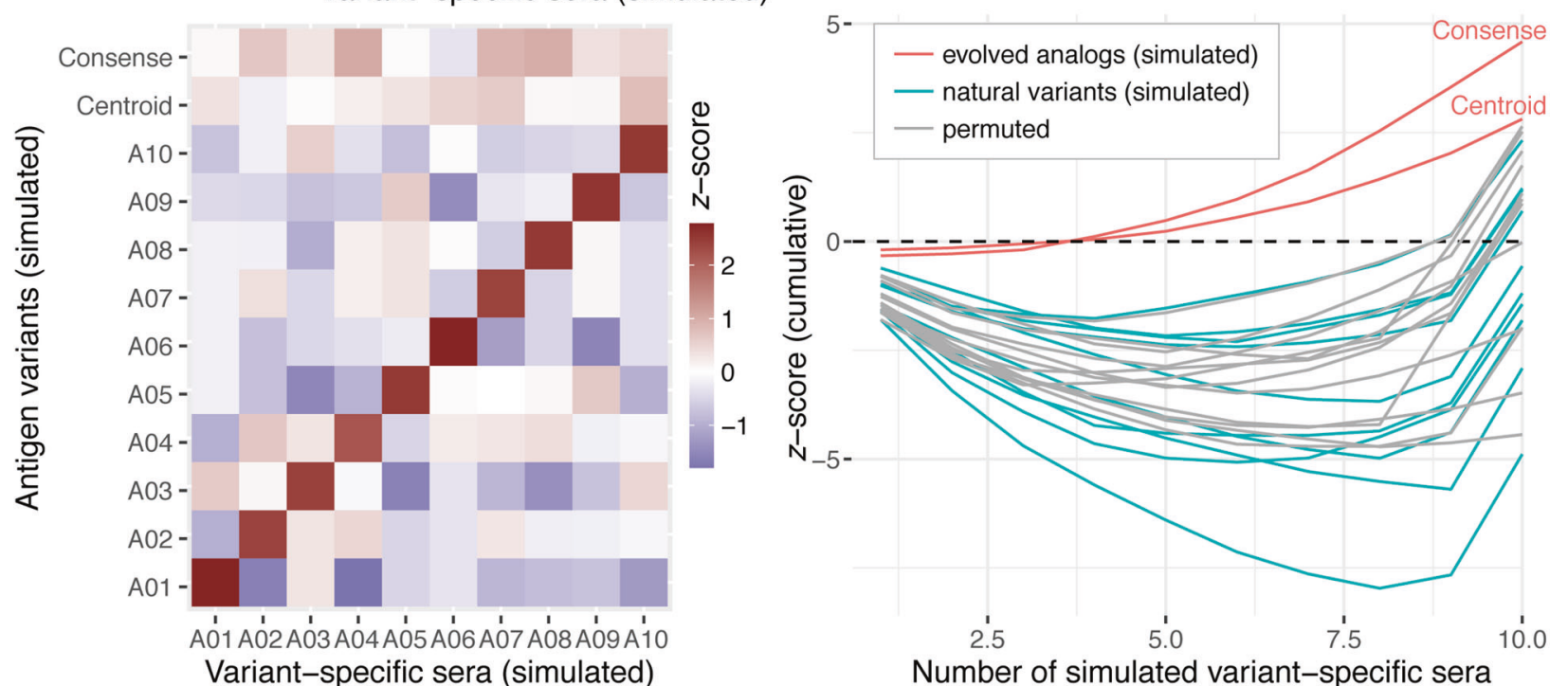

Fig. 8 Simulated antigen divergence and evolutionary analogs. The simulated population initially contained ten antigen variants, each represented by a 20-bit long randomly generated binary string. Each bit represented a variable antigen site. Using a genetic algorithm (Supplementary Information Text S2), we created a simulated population consisting of alleles "A01" through "A10" with maximally divergent sequences (Table 2). Subsequently, a consensus sequence ("Consense") was created from majority bits at individual positions. The centroid sequence ("Centroid") was generated by minimizing maximum sequence differences to the natural variants using a second round of genetic algorithm. Antigenic reactivity of a simulated variant $i$ to a simulated variant $j$-specific serum was assumed to be proportional to the sequence similarity between the two variants. Levels of antigenic activity were normalized to $z$-scores with respect to each simulated variant-specific serum. (Top left) Each panel shows levels of reactivity ( $x$-axis) of a simulated variant to simulated variant-specific sera ( $y$-axis). (Top right) A neighbor-joining tree of simulated variants based on pairwise Hamming distances. (Bottom left) A heat map representation of the levels of antigenic activity between the simulated variants ( $y$-axis) and the simulated variant-specific sera ( $x$-axis). (Bottom right) Each line shows cumulative $z$-scores ( $y$-axis) of a simulated natural variant (cyan), an evolutionary analog (red), or a natural variant after one round of permutation of $z$-scores among the simulated natural variants (gray).

In the current study, we described three evolutionary algorithms, proposed a theoretical model, and presented the initial proof-of-concept results demonstrating the broad antigenicity of the evolutionary centroids. The broader antigenicity of the centroids relative to the natural OspC variants makes the centroids promising candidates for improved diagnostics for Lyme disease. For vaccine development, it is further necessary to quantify the immunogenicity and test the protective efficacy of the centroids after immunization of mice with the synthetic OspC variants. Reactivity of the centroid-specific antibodies would then be tested against diverse B. burgdorferi strains through, e.g., immunofluorescence assays of cells present in field-collected ticks. Indeed, experiments are under way in our labs to generate centroidspecific antibodies, measure their immunogenicity and bacterial neutralization capability, and evaluate vaccine efficacy by challenging centroid-immunized mice with infected ticks carrying diverse $B$. burgdorferi strains. If validated, the OspC centroids would constitute a novel class of Lyme disease vaccines for humans and animals. If used as reservoir-targeted vaccines [86], the centroid antigens have the potential to reduce spirochete 
loads in natural reservoir hosts by eliciting immunity against all Lyme pathogenic strains.

Vaccines based on centroid antigens would be similar to the COBRA (Computationally Optimized Broadly Reactive Antigen) vaccines against influenza viruses and the vaccine candidates against HIV-1 viruses based on the "center-of-tree" ancestral sequences [8,52]. All three approaches are based on principles of antigen evolution and use automated computational design. While the COBRA design is based on consensus sequences and the center-of-tree algorithm infers ancestral sequences, the centroid design uses genetic algorithms to minimize sequence differences to the natural antigen variants. In the present study, the centroid algorithm was more effective than the consensus and root algorithms in broadening OspC cross-reactivity (Fig. 7). To date, the centroid algorithm did not enforce any structural constraints on the OspC molecule beyond the primary sequences. Additional functional and structural constraints to OspC diversification certainly exist. Indeed, the synthetic OspC centroids were less soluble than native variants under laboratory conditions, suggesting reduced structural stability of the synthetic OspC analogs. One approach of identifying additional constrains to OspC evolution is to develop a computational classifier by finetuning the pretrained universal protein models with a large number of sequences of natural OspC variants [87, 88]. Such an OspC-specific classifier should help identify centroids with improved functional and structural integrity while maintaining broad antigenicity.

Stable coexistence of antigen variants like OspC variants in $B$. burgdorferi is widespread in natural pathogen populations. The Dengue viral populations consist of four antigenically distinct serotypes associated with sequence variations of the envelop protein [89]. The influenza B viral populations contain two evolutionary lineages associated with sequence variations of hemagglutinin [90]. The malaria parasite populations are structured into antigenic groups associated with genetic variations of the var genes encoding an erythrocyte membrane protein [6]. If these pathogen strains indeed represent ecological niches shaped by host immunity $[3,91]$, evolutionary centroids would be a novel and effective strategy against a broad range of microbial pathogens.

\section{DATA AVAILABILITY}

All datasets are included in the Supplementary Information.

\section{CODE AVAILABILITY}

Source codes of the Perl and Python implementations of the centroid algorithm are available in a Github repository (https://github.com/weigangq/ag-div). Also available in the same Github repository are R scripts for generating the figures.

\section{REFERENCES}

1. Allen JA, Clarke BC. Frequency dependent selection: homage to E. B. Poulton. Biol J Linn Soc. 1984;23:15-8.

2. Papkou A, Guzella T, Yang W, Koepper S, Pees B, Schalkowski R, et al. The genomic basis of Red Queen dynamics during rapid reciprocal host-pathogen coevolution. Proc Natl Acad Sci USA. 2019;116:923-8.

3. Gupta S, Maiden MC, Feavers IM, Nee S, May RM, Anderson RM. The maintenance of strain structure in populations of recombining infectious agents. Nat Med. 1996;2:437-42.

4. Deitsch KW, Lukehart SA, Stringer JR. Common strategies for antigenic variation by bacterial, fungal and protozoan pathogens. Nat Rev Microbiol. 2009;7:493-503.

5. Ernst JD. Antigenic variation and immune escape in the MTBC. Adv Exp Med Biol. 2017;1019:171-90.

6. Pilosof S, He Q, Tiedje KE, Ruybal-Pesántez S, Day KP, Pascual M. Competition for hosts modulates vast antigenic diversity to generate persistent strain structure in Plasmodium falciparum. PLOS Biol. 2019;17:e3000336.

7. Ahmed Y, Tian M, Gao Y. Development of an anti-HIV vaccine eliciting broadly neutralizing antibodies. AIDS Res Ther. 2017;14:50.
8. Crevar CJ, Carter DM, Lee KYJ, Ross TM. Cocktail of H5N1 COBRA HA vaccines elicit protective antibodies against $\mathrm{H} 5 \mathrm{~N} 1$ viruses from multiple clades. Hum Vaccines Immunother. 2015;11:572-83.

9. Houser K, Subbarao K. Influenza vaccines: challenges and solutions. Cell Host Microbe. 2015;17:295-300.

10. Schwartz AM, Hinckley AF, Mead PS, Hook SA, Kugeler KJ. Surveillance for Lyme disease-United States, 2008-2015. Morb Mortal Wkly Rep Surveill Summ Wash DC 2002. 2017;66:1-12.

11. Adeolu M, Gupta RS. A phylogenomic and molecular marker based proposal for the division of the genus Borrelia into two genera: the emended genus Borrelia containing only the members of the relapsing fever Borrelia, and the genus Borreliella gen. nov. containing the members of the Lyme disease Borrelia (Borrelia burgdorferi sensu lato complex). Antonie Van Leeuwenhoek. 2014;105:1049-72.

12. Margos G, Marosevic D, Cutler S, Derdakova M, Diuk-Wasser M, Emler S, et al. There is inadequate evidence to support the division of the genus Borrelia. Int J Syst Evol Microbiol. 2017;67:1081-4.

13. Casjens S, Palmer N, Van Vugt R, Mun Huang W, Stevenson B, Rosa P, et al. A bacterial genome in flux: the twelve linear and nine circular extrachromosomal DNAs in an infectious isolate of the Lyme disease spirochete Borrelia burgdorferi. Mol Microbiol. 2000;35:490-516.

14. Mongodin EF, Casjens SR, Bruno JF, Xu Y, Drabek EF, Riley DR, et al. Inter- and intra-specific pan-genomes of Borrelia burgdorferi sensu lato: genome stability and adaptive radiation. BMC Genom. 2013;14:693.

15. Tilly K, Bestor A, Rosa PA. Lipoprotein succession in Borrelia burgdorferi: similar but distinct roles for OspC and VlsE at different stages of mammalian infection. Mol Microbiol. 2013;89:216-27.

16. Aslam B, Nisar MA, Khurshid M, Farooq, Salamat MK. Immune escape strategies of Borrelia burgdorferi. Future Microbiol. 2017;12:1219-37.

17. Coutte L, Botkin DJ, Gao L, Norris SJ. Detailed analysis of sequence changes occurring during vlsE antigenic variation in the mouse model of Borrelia burgdorferi infection. PLoS Pathog. 2009;5:e1000293.

18. Jacquot $M$, Gonnet $M$, Ferquel $E$, Abrial D, Claude A, Gasqui $P$, et al. Comparative population genomics of the Borrelia burgdorferi species complex reveals high degree of genetic isolation among species and underscores benefits and constraints to studying intra-specific epidemiological processes. PLoS ONE. 2014;9:e94384.

19. Seifert SN, Khatchikian CE, Zhou W, Brisson D. Evolution and population genomics of the Lyme borreliosis pathogen, Borrelia burgdorferi. Trends Genet TIG. 2015;31:201-7.

20. Schwartz I, Margos G, Casjens SR, Qiu W-G, Eggers CH. Multipartite genome of Lyme disease Borrelia: Structure, Variation and Prophages. Curr Issues Mol Biol. 2020;42:409-54.

21. Pritt BS, Respicio-Kingry LB, Sloan LM, Schriefer ME, Replogle AJ, Bjork J, et al. Borrelia mayonii sp. nov., a member of the Borrelia burgdorferi sensu lato complex, detected in patients and ticks in the upper midwestern United States. Int J Syst Evol Microbiol. 2016;66:4878-80.

22. Wang I-N, Dykhuizen DE, Qiu W, Dunn JJ, Bosler EM, Luft BJ. Genetic diversity of ospC in a local population of Borrelia burgdorferi sensu stricto. Genetics. 1999; 151:15-30.

23. Di L, Wan Z, Akther S, Ying C, Larracuente A, Li L, et al. Genotyping and quantifying Lyme pathogen strains by deep sequencing of the outer surface protein C (ospC) locus. J Clin Microbiol. 2018;56:e00940-18.

24. Barbour AG, Travinsky B. Evolution and distribution of the ospC gene, a transferable serotype determinant of Borrelia burgdorferi. mBio. 2010;1:e00153-10.

25. Brisson D, Dykhuizen DE. ospC Diversity in Borrelia burgdorferi different hosts are different niches. Genetics. 2004;168:713-22.

26. Norris SJ. v/s antigenic variation systems of Lyme disease Borrelia: eluding host immunity through both random, segmental gene conversion and framework heterogeneity. Microbiol Spectr. 2014;2:MDNA3-0038-2014.

27. Lin T, Gao L, Edmondson DG, Jacobs MB, Philipp MT, Norris SJ. Central role of the Holliday junction helicase RuvAB in vlsE recombination and infectivity of Borrelia burgdorferi. PLoS Pathog. 2009;5:e1000679.

28. Zhang JR, Hardham JM, Barbour AG, Norris SJ. Antigenic variation in Lyme disease borreliae by promiscuous recombination of VMP-like sequence cassettes. Cell. 1997;89:275-85.

29. Glöckner G, Schulte-Spechtel U, Schilhabel M, Felder M, Sühnel J, Wilske B, et al. Comparative genome analysis: selection pressure on the Borrelia v/s cassettes is essential for infectivity. BMC Genom. 2006;7:211.

30. Graves CJ, Ros VID, Stevenson B, Sniegowski PD, Brisson D. Natural selection promotes antigenic evolvability. PLoS Pathog. 2013;9:e1003766.

31. Xu Q, McShan K, Liang FT. Essential protective role attributed to the surface lipoproteins of Borrelia burgdorferi against innate defences. Mol Microbiol. 2008;69:15-29.

32. Tilly K, Krum JG, Bestor A, Jewett MW, Grimm D, Bueschel D, et al. Borrelia burgdorferi OspC protein required exclusively in a crucial early stage of mammalian infection. Infect Immun. 2006;74:3554-64. 
33. Carrasco SE, Troxell B, Yang Y, Brandt SL, Li H, Sandusky GE, et al. Outer surface protein OspC is an antiphagocytic factor that protects Borrelia burgdorferi from phagocytosis by macrophages. Infect Immun. 2015;83:4848-60.

34. Önder Ö, Humphrey PT, McOmber B, Korobova F, Francella N, Greenbaum DC, et al. OspC is potent plasminogen receptor on surface of Borrelia burgdorferi. J Biol Chem. 2012;287:16860-8.

35. Wilske B, Preac-Mursic V, Jauris S, Hofmann A, Pradel I, Soutschek E, et al. Immunological and molecular polymorphisms of OspC, an immunodominant major outer surface protein of Borrelia burgdorferi. Infect Immun. 1993;61:2182-91.

36. Bockenstedt LK, Hodzic E, Feng S, Bourrel KW, de Silva A, Montgomery RR, et al. Borrelia burgdorferi strain-specific OspC-mediated immunity in mice. Infect Immun. 1997;65:4661-7.

37. Jacquet M, Durand J, Rais O, Voordouw MJ. Cross-reactive acquired immunity influences transmission success of the Lyme disease pathogen, Borrelia afzelii. Infect Genet Evol J Mol Epidemiol Evol Genet Infect Dis. 2015;36:131-40.

38. Melo R, Richer L, Johnson DL, Gomes-Solecki M. Oral immunization with OspC does not prevent tick-borne Borrelia burgdorferi infection. PloS ONE. 2016;11: e0151850.

39. Bhatia B, Hillman C, Carracoi V, Cheff BN, Tilly K, Rosa PA. Infection history of the blood-meal host dictates pathogenic potential of the Lyme disease spirochete within the feeding tick vector. PLOS Pathog. 2018;14:e1006959.

40. Haven J, Vargas LC, Mongodin EF, Xue V, Hernandez Y, Pagan P, et al. Pervasive recombination and sympatric genome diversification driven by frequencydependent selection in Borrelia burgdorferi, the Lyme disease bacterium. Genetics. 2011;189:951-66.

41. Rannala B, Qiu WG, Dykhuizen DE. Methods for estimating gene frequencies and detecting selection in bacterial populations. Genetics. 2000;155:499-508.

42. Qiu W-G, Dykhuizen DE, Acosta MS, Luft BJ. Geographic uniformity of the Lyme disease spirochete (Borrelia burgdorferi) and its shared history with tick vector (Ixodes scapularis) in the northeastern United States. Genetics. 2002;160:833-49.

43. Hoen AG, Margos G, Bent SJ, Diuk-Wasser MA, Barbour A, Kurtenbach K, et al. Phylogeography of Borrelia burgdorferi in the eastern United States reflects multiple independent Lyme disease emergence events. Proc Natl Acad Sci. 2009; 106:15013-8.

44. States SL, Brinkerhoff RJ, Carpi G, Steeves TK, Folsom-O'Keefe C, DeVeaux M, et al Lyme disease risk not amplified in a species-poor vertebrate community: similar Borrelia burgdorferi tick infection prevalence and OspC genotype frequencies. Infect Genet Evol J Mol Epidemiol Evol Genet Infect Dis. 2014;27:566-75.

45. Durand J, Herrmann C, Genné D, Sarr A, Gern L, Voordouw MJ. Multistrain Infections with Lyme Borreliosis Pathogens in the Tick Vector. Appl Environ Microbiol. 2017;83:e02552-16.

46. Izac JR, Camire AC, Earnhart CG, Embers ME, Funk RA, Breitschwerdt EB, et al Analysis of the antigenic determinants of the OspC protein of the Lyme disease spirochetes: evidence that the $\mathrm{C} 10$ motif is not immunodominant or required to elicit bactericidal antibody responses. Vaccine. 2019;37:2401-7.

47. Baum E, Randall AZ, Zeller M, Barbour AG. Inferring epitopes of a polymorphic antigen amidst broadly cross-reactive antibodies using protein microarrays: a study of OspC proteins of Borrelia burgdorferi. PLoS ONE. 2013;8:e67445.

48. Ivanova L, Christova I, Neves V, Aroso M, Meirelles L, Brisson D, et al. Comprehensive seroprofiling of sixteen $B$. burgdorferi OspC: implications for Lyme disease diagnostics design. Clin Immunol. 2009;132:393-400.

49. Oliver LD, Earnhart CG, Virginia-Rhodes D, Theisen M, Marconi RT. Antibody profiling of canine IgG responses to the OspC protein of the Lyme disease spirochetes supports a multivalent approach in vaccine and diagnostic assay development. Vet J. 2016;218:27-33.

50. Earnhart CG, Marconi RT. Construction and analysis of variants of a polyvalent Lyme disease vaccine: approaches for improving the immune response to chimeric vaccinogens. Vaccine. 2007;25:3419-27.

51. Durand J, Jacquet M, Paillard L, Rais O, Gern L, Voordouw MJ. Cross-immunity and community structure of a multiple-strain pathogen in the tick vector. Appl Environ Microbiol. 2015;81:7740-52.

52. Rolland M, Jensen MA, Nickle DC, Yan J, Learn GH, Heath L, et al. Reconstruction and function of ancestral center-of-tree human immunodeficiency virus type 1 proteins. J Virol. 2007;81:8507-14.

53. Walter KS, Carpi G, Evans BR, Caccone A, Diuk-Wasser MA. Vectors as epidemiological sentinels: patterns of within-tick Borrelia burgdorferi diversity. PLOS Pathog. 2016;12:e1005759.

54. Barbour AG, Cook VJ. Genotyping strains of Lyme disease agents directly from ticks, blood, or tissue. Borrelia burgdorferi. New York, NY: Humana Press; 2018. pp. 1-11.

55. Edgar RC. MUSCLE: multiple sequence alignment with high accuracy and high throughput. Nucleic Acids Res. 2004;32:1792-7.

56. Hernández $Y$, Bernstein R, Pagan P, Vargas L, McCaig W, Ramrattan G, et al. BpWrapper: BioPerl-based sequence and tree utilities for rapid prototyping of bioinformatics pipelines. BMC Bioinform. 2018;19:76.

57. Stajich JE. An introduction to BioPerl. Methods Mol Biol Clifton NJ. 2007:406:535-48.
58. Stamatakis A. RAxML version 8: a tool for phylogenetic analysis and post-analysis of large phylogenies. Bioinformatics. 2014;30:1312-3.

59. Fortin F-A, De Rainville F-M, Gardner M-A, Parizeau M, Gagné C. DEAP: evolutionary algorithms made easy. J Mach Learn Res. 2012;13:2171-5.

60. Krupka M, Masek J, Barkocziova L, Knotigova PT, Kulich P, Plockova J, et al. The position of His-tag in recombinant OspC and application of various adjuvants affects the intensity and quality of specific antibody response after immunization of experimental mice. PLOS ONE. 2016;11:e0148497.

61. Molins CR, Sexton C, Young JW, Ashton LV, Pappert R, Beard CB, et al. Collection and characterization of samples for establishment of a serum repository for Lyme disease diagnostic test development and evaluation. J Clin Microbiol. 2014;52:3755-62.

62. CDC. Recommendations for test performance and interpretation from the Second National Conference on Serologic Diagnosis of Lyme Disease. MMWR Morb Mortal Wkly Rep. 1995;44:590-1.

63. Ivanova LB, Tomova A, González-Acuña D, Murúa R, Moreno CX, Hernández $C$ et al. Borrelia chilensis, a new member of the Borrelia burgdorferi sensu lato complex that extends the range of this genospecies in the Southern Hemisphere. Environ Microbiol. 2013;16:1069-80.

64. Schindelin J, Rueden CT, Hiner MC, Eliceiri KW. The ImageJ ecosystem: An open platform for biomedical image analysis. Mol Reprod Dev. 2015;82:518-29.

65. Moritz CP, Tholance $Y$, Lassablière F, Camdessanché J-P, Antoine J-C. Reducing the risk of misdiagnosis of indirect ELISA by normalizing serum-specific background noise: The example of detecting anti-FGFR3 autoantibodies. J Immunol Methods. 2019;466:52-6.

66. Zweig MH, Campbell G. Receiver-operating characteristic (ROC) plots: a fundamental evaluation tool in clinical medicine. Clin Chem. 1993;39:561-77.

67. Scrucca L. GA: a package for genetic algorithms in. R J Stat Softw. 2013;53:1-37.

68. Chen J, Hermelin D, Sorge M. On computing centroids according to the $p$-norms of Hamming distance vectors. ArXiv. 2019. https://arxiv.org/abs/1807.06469.

69. Chen Z-Z, Ma B, Wang L. A three-string approach to the closest string problem. J Comput Syst Sci. 2012;78:164-78.

70. Georgieva M, Buckee CO, Lipsitch M. Models of immune selection for multi-locus antigenic diversity of pathogens. Nat Rev Immunol. 2019;19:55-62.

71. Kumaran D, Eswaramoorthy S, Luft BJ, Koide S, Dunn JJ, Lawson CL, et al. Crystal structure of outer surface protein $C(\mathrm{OspC})$ from the Lyme disease spirochete, Borrelia burgdorferi. EMBO J. 2001;20:971-8.

72. Izac JR, Marconi RT. Diversity of the Lyme disease spirochetes and its influence on immune responses to infection and vaccination. Vet Clin N Am Small Anim Pract. 2019:49:671-86.

73. Edmondson DG, Prabhakaran S, Norris SJ, Ullmann AJ, Piesman J, Dolan M, et al Enhanced protective immunogenicity of homodimeric Borrelia burgdorferi outer surface protein C. Clin Vaccine Immunol. 2017;24:e00306-16.

74. Gilmore RD, Mbow ML. Conformational nature of the Borrelia burgdorferi B31 outer surface protein C protective epitope. Infect Immun. 1999;67:5463-9.

75. Qiu W-G, Schutzer SE, Bruno JF, Attie O, Xu Y, Dunn JJ, et al. Genetic exchange and plasmid transfers in Borrelia burgdorferi sensu stricto revealed by three-way genome comparisons and multilocus sequence typing. Proc Natl Acad Sci USA. 2004;101:14150-5.

76. Zinder D, Bedford T, Gupta S, Pascual M. The roles of competition and mutation in shaping antigenic and genetic diversity in influenza. PLoS Pathog. 2013;9: e1003104.

77. Livey I, Gibbs CP, Schuster R, Dorner F. Evidence for lateral transfer and recombination in OspC variation in Lyme disease Borrelia. Mol Microbiol. 1995;18:257-69.

78. Waddell LA, Greig J, Mascarenhas M, Harding S, Lindsay R, Ogden N. The accuracy of diagnostic tests for Lyme disease in humans, a systematic review and meta-analysis of North American research. PloS ONE. 2016;11:e0168613.

79. Branda JA, Strle K, Nigrovic LE, Lantos PM, Lepore TJ, Damle NS, et al. Evaluation of modified 2-tiered serodiagnostic testing algorithms for early Lyme disease. Clin Infect Dis. 2017:64:1074-80.

80. Pegalajar-Jurado A, Schriefer ME, Welch RJ, Couturier MR, MacKenzie T, Clark RJ, et al. Evaluation of modified two-tiered testing algorithms for Lyme disease laboratory diagnosis using well-characterized serum samples. J Clin Microbiol. 2018;56:e01943-17.

81. Lathrop SL, Ball R, Haber P, Mootrey GT, Braun MM, Shadomy SV, et al. Adverse event reports following vaccination for Lyme disease: December 1998-July 2000. Vaccine. 2002;20:1603-8.

82. Richer LM, Brisson D, Melo R, Ostfeld RS, Zeidner N, Gomes-Solecki M. Reservoir targeted vaccine against Borrelia burgdorferi: a new strategy to prevent Lyme disease transmission. J Infect Dis. 2014;209:1972-80.

83. Embers ME, Narasimhan S. Vaccination against Lyme disease: past, present, and future. Front Cell Infect Microbiol. 2013;3:00006.

84. Earnhart CG, Buckles EL, Marconi RT. Development of an OspC-based tetravalent, recombinant, chimeric vaccinogen that elicits bactericidal antibody against diverse Lyme disease spirochete strains. Vaccine. 2007;25:466-80. 
464

85. Earnhart CG, Marconi RT. An octavalent Lyme disease vaccine induces antibodies that recognize all incorporated OspC type-specific sequences. Hum Vaccin. 2007; 3:281-9.

86. Schuijt TJ, Hovius JW, van der Poll T, van Dam AP, Fikrig E. Lyme borreliosis vaccination: the facts, the challenge, the future. Trends Parasitol. 2011;27:40-7.

87. Rives A, Meier J, Sercu T, Goyal S, Lin Z, Liu J, et al. Biological structure and function emerge from scaling unsupervised learning to 250 million protein sequences. Proc Natl Acad Sci. 2021;118:e2016239118.

88. Strodthoff N, Wagner P, Wenzel M, Samek W. UDSMProt: universal deep sequence models for protein classification. Bioinformatics. 2020;36:2401-9.

89. Chen R, Vasilakis N. Dengue-Quo tu et quo vadis? Viruses. 2011;3:1562-608.

90. van de Sandt $C E$, Bodewes R, Rimmelzwaan GF, de Vries RD. Influenza $B$ viruses: not to be discounted. Future Microbiol. 2015;10:1447-65.

91. Buckee CO, Recker M, Watkins ER, Gupta S. Role of stochastic processes in maintaining discrete strain structure in antigenically diverse pathogen populations. Proc Natl Acad Sci. 2011;108:15504-9.

\section{ACKNOWLEDGEMENTS}

This work was supported by the Public Health Service awards Al139782 (to WGQ) and Al072810, Al074092 and Al155211 (to MGS) from the National Institute of Allergy and Infectious Diseases (NIAID) of the National Institutes of Health $(\mathrm{NIH})$ of the United States of America. Additional funding includes the grant CK000107 (to MGS) from the US Centers for Disease Control and Prevention (CDC) and the award 7F2400001 (to SM) from the PhRMA Foundation. LD and SK are supported in part by the award EB030275 from the National Institute of Biomedical Imaging and Bioengineering (NIBIB, to Brian Zeglis and WGQ) of the National Institutes of Health (NIH). The content of this paper is solely the responsibility of the authors and does not necessarily represent the official views of NIAID, NIH, or CDC. SA is supported in part by the Doctoral Program in Biology of the City University of New York. We thank Dr Mirella Salvatore (Weil Cornell Medical College) for introducing us to the study of influenza B viruses. Dr Christopher Sexton and Dr Jeannine Petersen (Division of Vector-Borne Diseases, CDC) prepared a custom panel of human sera for this study. Roman Shimonov and Justin Hiraldo (both of Hunter College) digitalized immunoblot images. We thank three anonymous referees for careful reading and constructive critiques of the original draft.

\section{AUTHOR CONTRIBUTIONS}

Conceptualization, funding acquisition, and supervision: MGS, WQ. Model development: SA, BS, SM, and WQ. Experimental Investigations: LD, SA, LI, and BW. Software implementation: $L D, E B$. Data analysis: $L D, S A, E B, B S, L I$, and WQ. Writing-original draft: WQ. Writing—review and editing: $L D, S A, B S, E B, S M$, and MGS.

\section{COMPETING INTERESTS}

The following authors declare potential competing interests: MGS (patents) and WGQ (patents). All other authors declare no competing interests in relation to this study.

\section{ADDITIONAL INFORMATION}

Supplementary information The online version contains supplementary material available at https://doi.org/10.1038/s41396-021-01089-4

Correspondence and requests for materials should be addressed to W.-G.Q.

Reprints and permission information is available at http://www.nature.com/ reprints

Publisher's note Springer Nature remains neutral with regard to jurisdictional claims in published maps and institutional affiliations. 